\title{
ON THE INFLUENCE OF FRICTIONAL PARAMETERIZATION IN WIND-DRIVEN OCEAN CIRCULATION MODELS
}

\author{
CLAUS W. BÖNING * \\ Institut für Meereskunde an der Universität Kiel, Kiel (F.R.G.)
}

(Received January 30, 1985; revised July 11, 1985; accepted October 18, 1985)

\begin{abstract}
Böning, C.W., 1986. On the influence of frictional parameterization in wind-driven ocean circulation models. Dyn. Atmos. Oceans, 10: 63-92.
\end{abstract}

In a series of numerical experiments the wind-driven ocean circulation is studied in an idealized, rectangular model ocean, which is forced by steady zonal winds and damped by lateral and/or bottom friction. The problem as described by the barotropic vorticity equation is characterized by a Rossby number $(R)$ and horizontal and/or vertical Ekman numbers $\left(E_{\mathrm{L}}, E_{\mathrm{B}}\right)$ only.

With free-slip conditions at the boundaries steady solutions for all chosen values of $R$ are obtained, provided the diffusivity is sufficiently large. For both the forms of frictional parameterization a northern boundary current emerges with an eastward penetration scale depending on $R$. The recirculation pattern in the oceanically relevant 'intermediate' range of $R$ is strongly affected by the type of friction. If lateral diffusion dominates bottom friction. a strong recirculating sub-gyre emerges in the northwestern corner of the basin. Its shape resembles the vertically integrated transport fields in recent eddy resolving model (EGCM) studies. The maximum transport is increased to values several times larger than the Sverdrup transport. The increase in transport is coupled with a development of closed contours of potential vorticity, enabling a nearly free inertial flow.

This behaviour provides a sharp contrast to the bottom friction case (Veronis) where inertial recirculation only takes place with values of $R$ so large that the eastward jet reaches the eastern boundary. It is shown that the linear friction law puts a strong constraint on the flow by preventing an intense recirculation in a small part of the basin.

A reduction of the diffusivity $\left(E_{\mathrm{L}}\right)$ in the lateral friction case leads to quasi-steady solutions. The interaction with eddies becomes an integral part of the time mean energetics but does not influence the recirculation character of the flow.

The main conclusion of the study is that the horizontal structure of the EGCM-transport fields can be explained in terms of a steady barotropic model where lateral friction represents the dominant dissipation mechanism.

\footnotetext{
* Present affiliation: Geophysical Fluid Dynamics Program, Princeton University, Princeton, NJ 08540, U.S.A.
} 


\section{INTRODUCTION}

There has been a long history of work on the problem of the wind-driven ocean circulation. Since the early papers of Stommel (1948) and Munk (1950) a particular class of models deals with the question of how the ocean circulation equilibrates in a simple, closed and often rectangular basin in response to a simple, steady windstress pattern, i.e., which processes are responsible for the horizontal structure of the wind-driven gyres. Under the heading 'Stommel-type' we can follow the line from the simple, linear one-layer models as cited above, which have shown the central role of the planetary vorticity gradient in establishing the westward intensification of the wind-gyres, to the increasingly complex models, i.e., two- or more layer eddy-resolving general circulation models (EGCMs) which are concerned with the role of spontaneously growing transients in the solutions-regarded as model analogues of real ocean eddies (e.g., Robinson et al., 1979; Holland et al., 1983).

Despite the relative simplicity of all these 'idealized' models it soon becomes a very difficult task to analyse their results and to understand their physics, e.g., the effect of the mesoscale variability on the time mean circulation in the EGCMs. Recently Harrison and Stalos (1982), Marshall (1984) and Kamenkovich et al. (1985) began to reconsider the barotropic problem. Because the mean flow upper layer dynamics in adiabatic, quasigeostrophic systems is essentially established by the solution of the barotropic vorticity equation (Harrison, 1982), the barotropic system may serve as a necessary and helpful basis to understand the more complex EGCM-flows.

The most serious deficiency in constructing idealized models is due to the fact that valid approximations to the full set of equations are not known. In connection with the wind-driven ocean problem this especially holds for the proper modelling of the vorticity sink, in other words the form of the frictional parameterization, and, if needed, the type of the dynamical boundary conditions. A common way to overcome such difficulties is to examine the influence of plausibly formulated parameterizations and chosen parameter values on the model solutions and to compare the model flow with the real ocean flow characteristics to eventyally justify the model by a posteriori success (rather than by a priori proof). However, this has only been done partly so far with regard to the wind-driven ocean circulation. Stated in the form of the barotropic vorticity equation, the problem is characterized by two external parameters only: a Rossby number $(R)$ and a horizontal $\left(E_{\mathrm{L}}\right)$ or vertical $\left(E_{\mathrm{B}}\right)$ Ekman number. Although some properties of the flow field may be explained in terms of simple-scale arguments (e.g., Niiler (1966) and Harrison and Stalos (1982)), the complete nonlinear, 
diffusive problem can be solved by numerical integration only. Independent of the frictional parameterization all the model flows show common features for low $R$ : (considering anticyclonic gyres) a northward shift of the gyre centres compared to the symmetrical linear flow, coupled with some reduction of the maximum transport of the western boundary current below the Sverdrup value.

However, the model results greatly differ when $R$ is increased to values where the inertial boundary layer width $\left(\delta_{I}\right)$ becomes comparable to, or even larger than, the corresponding frictional scale $\left(\delta_{\mathrm{L}}, \delta_{\mathrm{B}}\right)$. A model with lateral friction and no-slip boundary conditions does not allow steady solutions due to shear flow instabilities at the western wall (Bryan, 1963), whereas a bottom friction model (Veronis, 1966b) always reaches a steady state, establishing an eastward jet at the northern boundary. With large values of $R\left(\delta_{1}\right.$ three or four times $\left.\delta_{\mathrm{B}}\right)$ the eastward jet reaches the eastern wall. An intense recirculation builds up, which leads to an increase in maximum transport due to a southward advection of negative vorticity supporting the action of the wind field (Fig. 1). Harrison and Stalos (1982) re-examined the bottom friction model by analysing the equilibrium vorticity balances in various regions of the basin and the dependency on both single and double gyre wind forcing. The lateral friction/no-slip case was recently reconsidered by Kamenkovich et al. (1985) to rationalize the transient solutions.

It was shown in a series of experiments by Blandford (1971) that the difference in model behaviour critically depends on the imposed dynamical boundary conditions. In his bottom friction model-with a small lateral friction added - the flow changed from Veronis' northern boundary current case to an unsteady Bryan case with no eastward jet when changing the boundary conditions from free-slip to no-slip. This is supplemented by the lateral friction/free-slip model of Briggs (1980) where steady solutions emerged for all values of $R$, approaching a 'wind-modified Fofonoff-mode', symmetrical to the mid-basin. However, the oceanic relevance of this model is somewhat obscure as Briggs concentrated on the limit of very large values of $R$.

Marshall (1984) extended the single, steady gyre barotropic calculations to include a dynamically active internal jet partitioning counterrotating gyres. Using bottom plus biharmonic friction in connection with (unconventional) boundary conditions on the vorticity gradient ensuring no net dissipation of vorticity through the walls, a barotropic instability of the jet was maximized to transfer all the vorticity required to maintain the time-mean gyres. After Marshall and Shutts (1981), the sense of the eddy flux of vorticity was rationalized with reference to the eddy enstrophy equation into two contributions: a non-divergent flux responding to flow advection of eddy enstrophy and a divergent flux responding to the dissipation of eddy enstrophy 

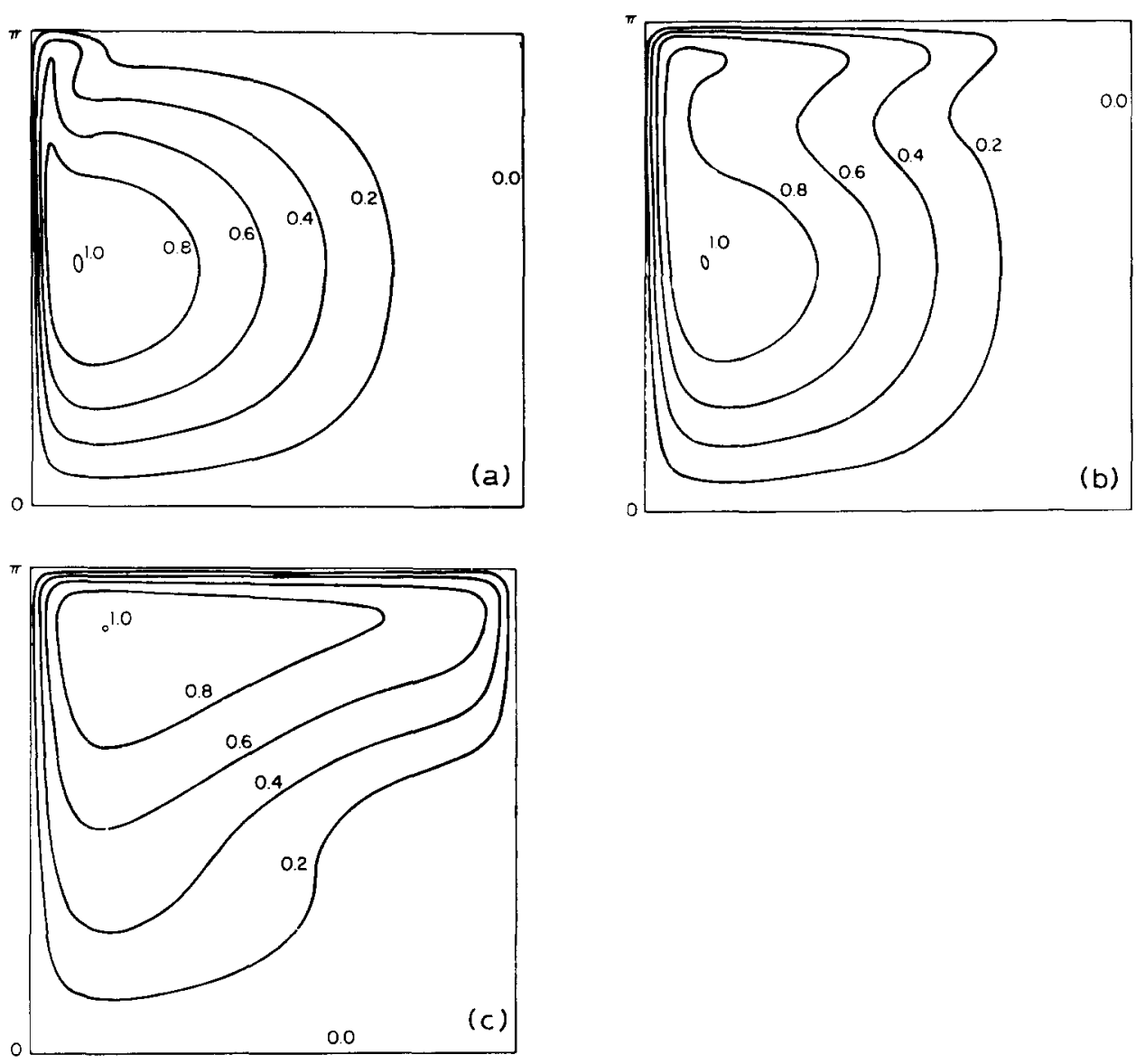

Fig. 1. Steady state streamfunction contours from the barotropic, bottom friction model of Veronis (1966). For the case (a) $\delta_{1} / \delta_{\mathrm{B}}=1.0$, (b) $\delta_{\mathrm{I}} / \delta_{\mathrm{B}}=2.0$, (c) $\delta_{\mathrm{I}} / \delta_{\mathrm{B}}=4.0$. The streamfunction is normalized with respect to its maximum value.

in the enstrophy cascade. The latter was shown to point systematically down the mean vorticity gradient suggesting a local applicability of a down-gradient flux parameterization, i.e., a lateral friction hypothesis.

Let us compare the prototype barotropic model results (Fig. 1) with a typical time mean horizontal transport pattern of an EGCM calculation (Fig. 2). Like the example shown in most EGCM studies free-slip conditions are used (to avoid the additional complication of resolving high shear zones at the walls) and, therefore, eastward jets are feasible. Compared with the barotropic bottom friction model the essential difference lies in the strong recirculation pattern with a subsequent increase in transport which occurs even if the penetration scale of the jet is far smaller than the basin width. Certainly, this seems to be an oceanographically relevant feature when 


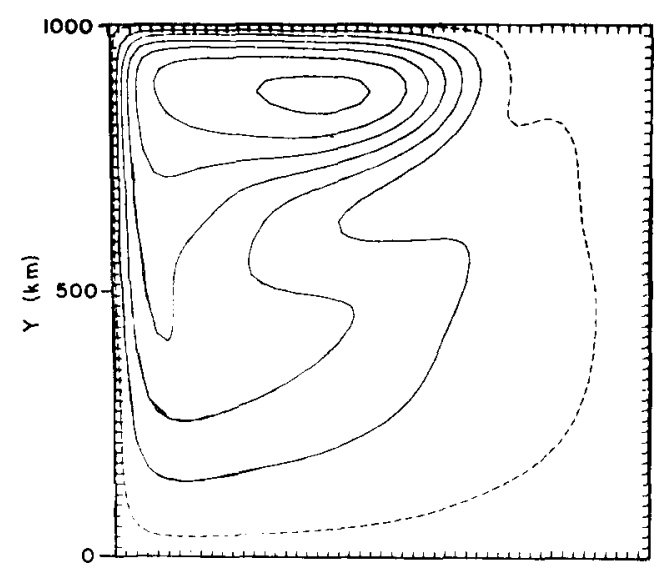

Fig. 2. Mean upper layer streamfunction $\psi$ of Holland's EGCM-experiment 1 (Holland. 1978). In our notation, $\delta_{\mathrm{I}} / \delta_{\mathrm{L}}=1.1$.

regarding Worthington's (1976) observations of a tight recirculation pattern directly south and east of the Gulf Stream. The recirculation strength is of the order of $90 \mathrm{~Sv}$ or about three times the transport of the Florida Current (Schmitz, 1980). Because, theoretically, such a volume of mass flow cannot be driven by the North Atlantic wind systems (Leetmaa and Bunker, 1978). its dynamical cause is thought to be the eddy field in the Gulf Stream system.

What we will show in this paper is that the same recirculation characteristics as in the EGCMs can be reproduced in a barotropic model with steady solutions. Essential in this sense is the parametrization of the vorticity sink. We will concentrate on a lateral friction model with free-slip boundary conditions which - in the oceanically relevant parameter regime of moderate non-linearity-has not been considered as yet.

\section{MODEL FORMULATION AND BACKGROUND}

We are concerned with the horizontal transport in a closed square-ocean basin of width $\pi L$ and constant depth $H$ on a mid-latitude $\beta$-plane. If lateral friction is included by a simple eddy viscosity hypothesis (Munk, 1950) and vertical friction by means of Ekman layers at both the top and the bottom. the model is stated in terms of the barotropic vorticity equation (BVE)

$\frac{\partial}{\partial t} \nabla^{2} \psi+J\left(\psi, \nabla^{2} \psi\right)+\beta \psi_{x}=\frac{1}{H} \operatorname{curl}_{z} \vec{\tau}+A \nabla^{4} \psi-K \nabla^{2} \psi$

governing the stream function $\psi$ for the vertically averaged velocity components $u=-\partial \psi / \partial y, v=\partial \psi / \partial x$. 
It is convenient to introduce a minimum number of external parameters and to study the dependence on these parameters. Discussing the problem in the non-dimensional form

$\frac{\partial}{\partial t} \nabla^{2} \psi^{\prime}+R J\left(\psi^{\prime}, \nabla^{2} \psi^{\prime}\right)+\psi_{x}^{\prime}=\operatorname{curl}_{z} \vec{\tau}+E_{\mathrm{L}} \nabla^{4} \psi^{\prime}-E_{\mathrm{B}} \nabla^{2} \psi^{\prime}$

these are an external (basin) Rossby number $R$ and horizontal and vertical Ekman numbers $E_{\mathrm{L}}$ and $E_{\mathrm{B}}$, defined to be

$R=\frac{T_{0}}{H \beta^{2} L^{3}} \quad E_{\mathrm{L}}=\frac{A}{\beta L^{3}} \quad E_{\mathrm{B}}=\frac{K}{\beta L}$

if the non-dimensionalization is accomplished by the scaling $(x, y)=L\left(x^{\prime}, y^{\prime}\right) \quad t=(\beta L)^{-1} t^{\prime} \quad \tau=T_{0} \tau^{\prime} \quad \psi=T_{0} /(\beta H)^{\psi^{\prime}}$

In the following we will drop the primes. Generally, we seek solutions of eq. 2 in the square $(\pi, \pi)$-basin with the boundary conditions

$\psi=0 \quad$ and $\quad \frac{\partial^{2} \psi}{\partial n^{2}}=0$

where $n$ is the local, normal coordinate, subject to a steady, anticyclonic, single-gyre forcing pattern

$\operatorname{curl}_{z} \tau^{\prime}=-\sin y^{\prime}$

For $R, E_{\mathrm{L}}, E_{\mathrm{B}} \ll 1$ we assume that the Sverdrup-balance will hold in most parts of the basin, and we can define the western boundary layer scales

$\delta_{\mathrm{I}}=R^{1 / 2} \quad \delta_{\mathrm{L}}=E_{\mathrm{L}}^{1 / 3} \quad \delta_{\mathrm{B}}=E_{\mathrm{B}}$

supposing the boundary layers are principally controlled by either the inertial term or by one of the frictional terms. Let the width of the ocean's western boundary currents be $1 \%$ of the width of the oceans and assume that both frictional and non-linear processes are important for scales comparable to this lateral scale. Then we would expect

$\delta_{\mathrm{I}} \sim\left(\delta_{\mathrm{L}}\right.$ or $\left.\delta_{\mathrm{B}}\right) \sim \pi / 100$

or

$R \sim 1 \cdot 10^{-3} \quad E_{\mathrm{L}} \sim 3 \cdot 10^{-5} \quad E_{\mathrm{B}} \sim 3 \cdot 10^{-2}$

to be the relevant magnitudes of the model parameters. However, in connection with the finite resolution in a numerical model one is generally confined to a minimum value of the Ekman number depending on the computer time available. 
Equation 2.2 is integrated numerically by using the spectral approximation technique. Thus, the spatial dependence of the inhomogeneous term and of the dependent variable in the BVE is represented by a truncated series of orthogonal functions, and a system of ordinary differential equations for the time-dependent expansion coefficients is constructed by the Galerkin approximation. We will not discuss details here; comprehensive reviews of the spectral method and its general properties may be found in Machenhauer (1979) and Gottlieb and Orszag (1977). Recently, several authors have adopted various spectral expansion schemes for the numerical modelling of oceanic systems, e.g., Haidvogel et al. (1980) for barotropic open ocean studies and Krauss and Wübber (1982a,b) for linear models of wind-driven flows in a stratified ocean.

To account for the boundary conditions (2.3) we approximate the fields by a double Fourier-sine-series

$\operatorname{curl}_{z} \tau(x, y) \sim \sum_{m=1}^{N} \sum_{n=1}^{N} T_{m, n} \sin m x \sin n y$

$\psi(x, y, t) \sim \sum_{m=1}^{N} \sum_{n=1}^{N} \psi_{m, n}(t) \sin m x \sin n y$

This approach was made in a series of WDOC-studies by Veronis (1963, $1966 \mathrm{c}$ ), but was confined to a very limited number of Fourier components due to the expensive computations of the convolution sums of the non-linear terms. This deficiency can be overcome by means of the so-called pseudospectral method for the calculation of non-linear terms as originally suggested by Orszag (1969). Thus, the multiplications involved in the Jacobian term are not evaluated in the spectral domain but rather on an equivalent grid in the physical domain.

The truncated spectral equations for the expansion coefficients $\psi_{m, n}(t)$ are advanced in time by using a leapfrog scheme (except for the viscous terms) yielding

$$
\begin{aligned}
\psi_{m, n}^{K+1}= & \psi_{m, n}^{K-1}+r\left\{T_{m, n}-R J\left(\psi^{K}, \zeta^{K}\right)-\sum_{j=1}^{N} j a_{m, j} \psi_{j, n}^{K}\right\} \\
& -r\left\{\left(m^{2}+n^{2}\right) E_{\mathrm{L}} \psi_{m, n}^{K-1}+E_{\mathrm{B}} \psi_{m, n}^{K-1}\right\} \text { for } m, n=1,2, \ldots N
\end{aligned}
$$

where

$$
r=2 \Delta t /\left(m^{2}+n^{2}\right) \quad a_{m, j}=4 m /\left(\left(m^{2}-j^{2}\right) \pi\right)
$$


and the Jacobian term is treated as mentioned above, employing the energyconserving form (Arakawa, 1966)

$J(\psi, \zeta)=\left(\psi_{x}, \zeta\right)_{y}-\left(\psi_{y}, \zeta\right)_{x}$

More details of the numerical approach are presented in Böning (1985). The most serious problem of the expansion in a sum of sine terms is due to the $\beta \psi_{x}$ term in the vorticity equation causing a non-uniform convergence of the scheme. Preliminary tests were made by varying the number $N$ of the expansion coefficients to determine the necessary values that yield reliable results for specific choices of the external parameters $E_{\mathrm{B}}, E_{\mathrm{L}}, R$. The resulting criterion is equivalent to that of finite-difference models, e.g., Veronis (1966b): In general, no alterations of the solutions could be stated, provided $N$ is large enough so that there was at least one point of the equivalent grid in the boundary layer, where it was sufficient to resolve the largest of $\delta_{\mathrm{B}}, \delta_{\mathrm{L}}, \delta_{\mathrm{I}}$. On the other hand a significant reduction of $N$ below this threshold value resulted in spatial oscillations in the stream-function and especially in the vorticity fields as symptomatic of a Gibb's phenomenon. For most of the calculations yielding steady solutions, $31 \times 31$ wave numbers proved to be sufficient, whereas up to $63 \times 63$ wave numbers were required to accomodate for a significant reduction in the friction for quasi-steady cases. The programmes were tested by reproducing some of the lateral friction/free-slip model results of Briggs (1980); we found a good agreement with his results.

\section{CALCULATiONS}

The experiments are arranged in three sequences to represent the model behaviour in the $\left(E_{\mathrm{L}}, E_{\mathrm{B}}, R\right)$-parameter space. Contour maps show the streamfunction $\psi$, the relative vorticity $\zeta$ and the potential vorticity $q=\xi+f$ in the state of equilibrium. Contour lines of $\psi$ are drawn with a fixed interval in each sequence so that changes in the maximum values and-consequently - in the maximum transports are visible from changes in the number of isolines. Additionally, the values of the maximum transport are presented in dimensional units. Therefore, we shall use the following basin parameters during the whole paper: a basin width $\pi L=2 \cdot 10^{8} \mathrm{~cm}$, an amplitude of the windstress $T_{0}=2 \mathrm{~cm}^{2} \mathrm{~s}^{-2}$ and a planetary vorticity gradient $\beta=2 \cdot 10^{-13} \mathrm{~cm}^{-1} \mathrm{~s}^{-1}$. By means of this forcing the linear, frictionless Sverdrup transport in our quadratic basin amounts to $T_{0} \pi / \beta=31.4 \cdot 10^{12}$ $\mathrm{cm}^{3} \mathrm{~s}^{-1}$, which may serve as a reference to the maximum transports such as computed numerically in the advective-diffusive model. (For a quantitative comparison with the results of other studies note further that some authors used curl $\tau=-\sin x \sin y$ instead of curl $\tau=-\sin y$ as the forcing 
function which does not matter for the solution patterns but is essential for their amplitudes.)

\subsection{Lateral friction model}

Our first task is to complete the picture of the lateral friction/free-slip model behaviour in the intermediate range between weakly non-linear and strongly non-linear flows. Table I presents a sequence of experiments with an increasing Rossby number $R$. A weakly non-linear case is shown in Fig. 3. Besides the frictional recirculation at the outer edge of the western boundary current (Munk's return flow), the flow in this parameter range behaves just the same as in the bottom friction model (BFM) (Veronis, $1966 \mathrm{a}, \mathrm{b})$. Owing to the northward advection of negative vorticity in the boundary layer, which counteracts the effect of the windstress in the Sverdrup interior, the transport in the non-linear case is slightly reduced. The relative vorticity is almost zero in the interior and is concentrated near the western wall, but even there it is not able to distort the dominance of the planetary vorticity gradient on the potential vorticity contours.

The situation changes when non-linear effects become more prominent (Figs. 4 and 5). Indeed, an eastward jet, such as in the BFM, builds up at the northern boundary accompanied by a recirculating flow which passes a region of positive vorticity before entering the Sverdrup interior. Simultaneously, closed streamlines appear in the northwestern corner trapping fluid particles which never go through the Sverdrup regime. The maximum transport shows a significant increase. These features are still more pronounced in Fig. 6 where the inertial and frictional scales-which, of course, have lost much of their relevance at this stage-are equivalent.

TABLE I

External parameters and maximum gyre transports of the experiments with lateral friction only

\begin{tabular}{llllc}
\hline Experiment & $E_{\mathrm{L}}$ & $R$ & $\delta_{\mathrm{I}} / \delta_{\mathrm{L}}$ & $\begin{array}{c}\text { Transport } \\
\left(10^{12} \mathrm{~cm}^{3} \mathrm{~s}^{-1}\right)\end{array}$ \\
\hline 1 & $9 \cdot 10^{-4}$ & 0 & 0 & 38.2 \\
2 & $9 \cdot 10^{-4}$ & $3.72 \cdot 10^{-4}$ & 0.2 & 38.1 \\
3 & $9 \cdot 10^{-4}$ & $2.33 \cdot 10^{-3}$ & 0.5 & 34.6 \\
4 & $9 \cdot 10^{-4}$ & $5.96 \cdot 10^{-3}$ & 0.8 & 54.1 \\
5 & $9 \cdot 10^{-4}$ & $9.31 \cdot 10^{-3}$ & 1.0 & 89.2 \\
6 & $9 \cdot 10^{-4}$ & $2.10 \cdot 10^{-2}$ & 1.5 & $425^{\mathrm{a}}$ \\
\hline
\end{tabular}

a The kinetic energy of the flow is still increasing at 200 days of integration. 

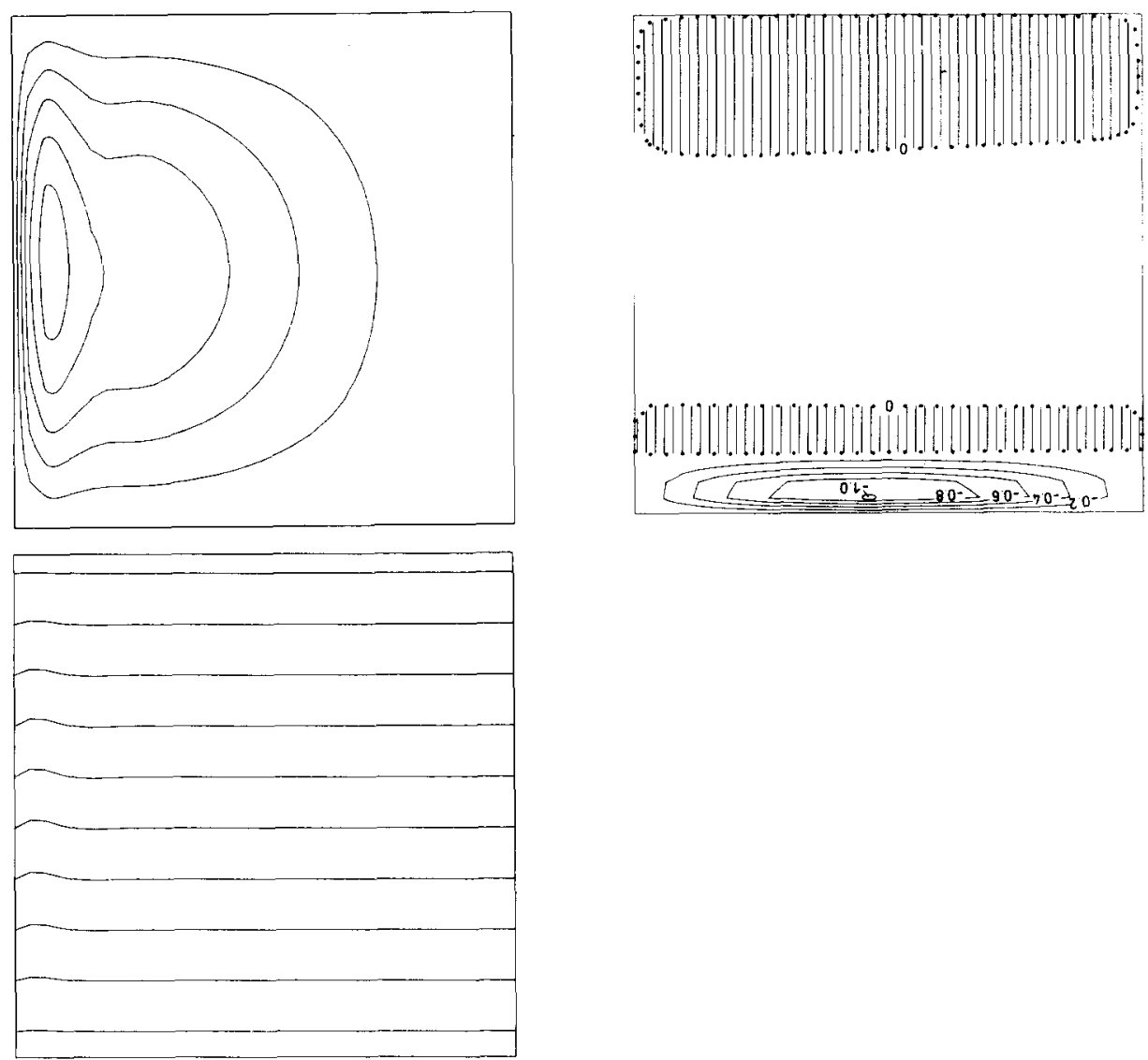

Fig. 3. Contours of $\psi, \zeta, q$ (in that order reading down) for experiment $2, \delta_{1} / \delta_{\mathrm{L}}=0.2$. $\psi_{\max }=3.46, \zeta_{\max }=140.3$; contour intervals $\Delta \psi=0.69, \Delta q=0.4 \cdot 10^{-5} \mathrm{~s}^{-1}$. The relative vorticity is normalized with respect to its maximum value. Regions of positive $\zeta$ are shaded.

The relative vorticity maps reveal a broadening and intensifying region of negative vorticity in the north. Its edge divides the trapped flow from the one turning southeastward to re-enter the Sverdrup interior. The presence of a strong recirculating sub-gyre provides a sharp contrast to BFM-flows. There, a closed inertial recirculation only exists when the flow becomes strongly non-linear and the eastward jet crosses the whole basin; southward advection of negative vorticity, which acts in the same sense as the wind and results in an increase in transport, only takes place at the eastern boundary.

The potential vorticity contours are strongly deformed reflecting the intense relative vorticity in the northwestern corner. The isolines of $q-$ called geostrophic contours or isostrophes-may serve as an alternative aid to 

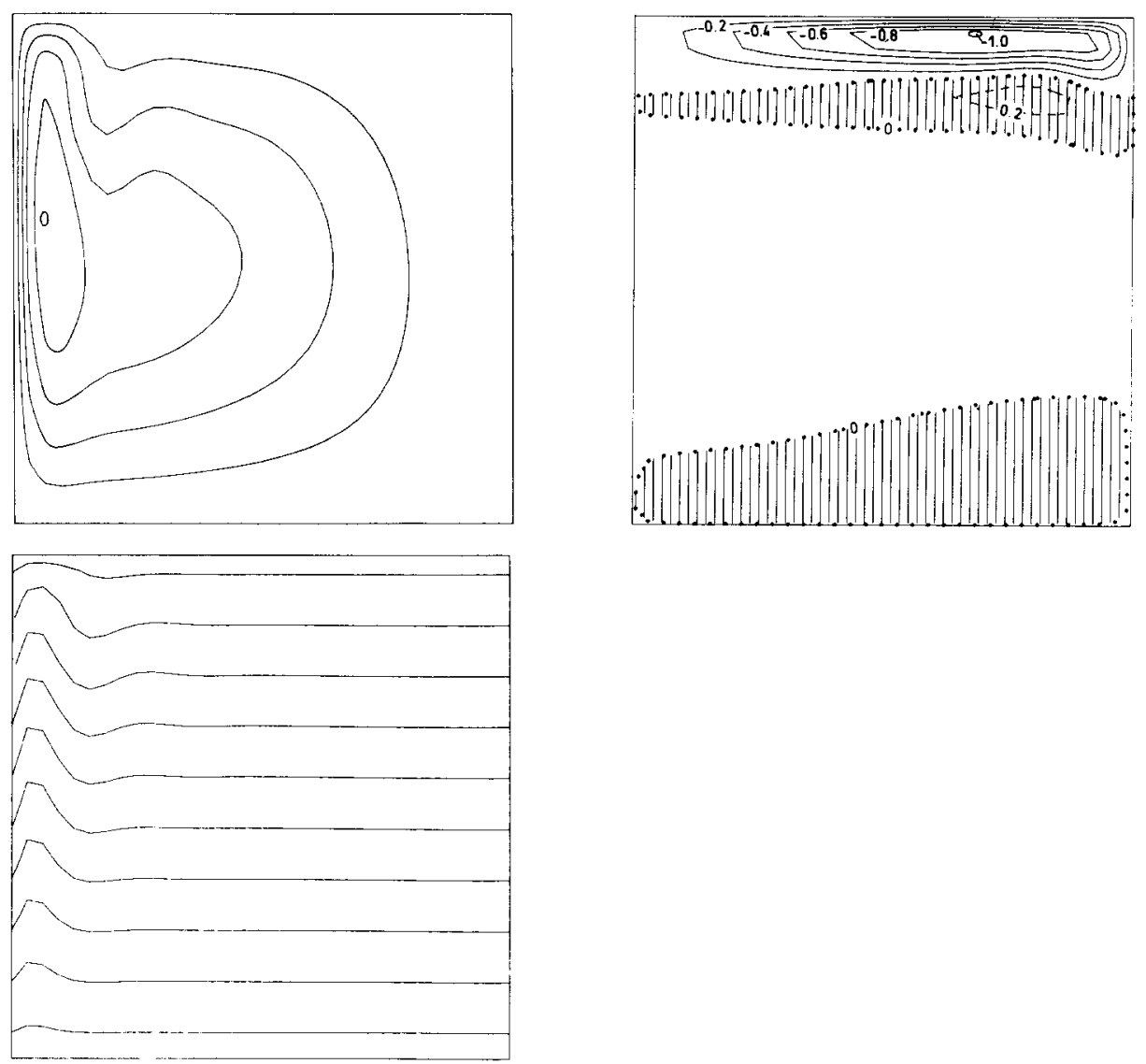

Fig. 4. $\psi, \zeta, q$ for experiment $3, \delta_{\mathrm{I}} / \delta_{\mathrm{L}}=0.5 . \psi_{\max }=3.81,\left|\zeta_{\max }\right|=131.5$. Contour intervals as in Fig. 3.

discuss the significant changes in transport. Writing the vorticity equation as $\frac{\mathrm{d} q}{\mathrm{~d} t}=$ forcing $f+$ dissipation $d$

it is evident that fluid particles can only cross isostrophes at a rate which the external forces $f$ or $d$ can account for. This limits the circulation in the interior where the geostrophic contours are blocked by the basin walls. The Sverdrup constraint is broken for trajectories involving no changes in $q$. The sub-gyre flow with streamlines almost parallel to the geostrophic contours is able to circulate nearly free of any influence by external forces. A more thorough discussion of the vorticity balance in the recirculation regime will be given in section 5 .

Figure 7 presents a case where the inertial scale is 1.5 times the diffusive scale. The flow has become essentially non-linear and tends to be like the 

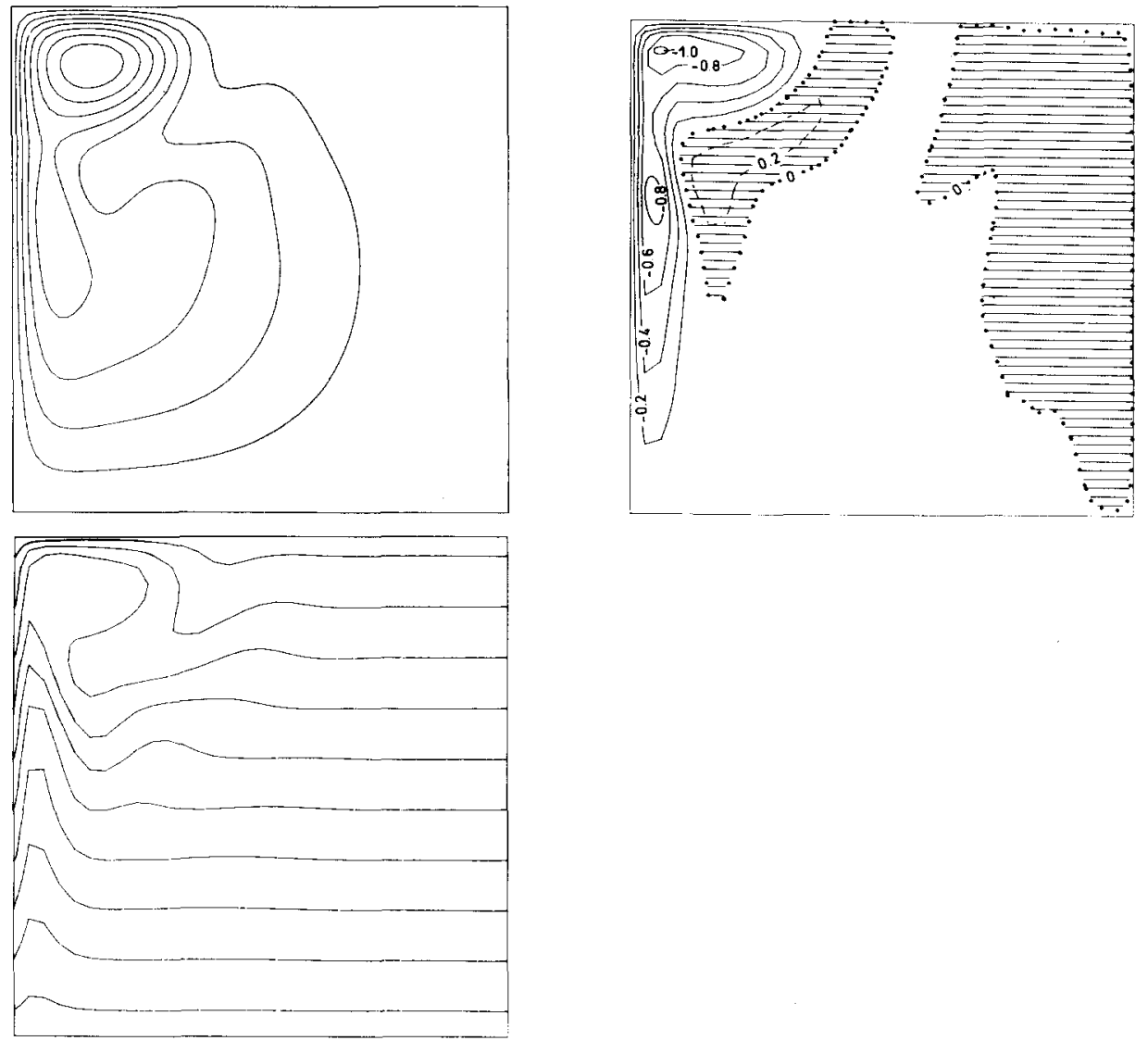

Fig. 5. $\psi, \zeta, q$ for experiment $4, \delta_{1} / \delta_{\mathrm{L}}=0.8 . \psi_{\max }=5.34,\left|\zeta_{\max }\right|=130.8$. Contour intervals as in Fig. 3.

symmetric flow-field results of Briggs (1980). Briggs demonstrated that the solution in the limit of high $R$ can be interpreted as an inertial Fofonoff-gyre (Fofonoff, 1954) obeying

$J\left(\psi, \nabla^{2} \psi\right)=0$

with the additional balance of $f$ and $d$

$-E_{\mathrm{L}} \nabla^{4} \psi=\operatorname{curl}_{z} \tau$

Yet, it must be mentioned that the situation does not change with respect to a sinusoidal wind field if a bottom friction model is considered; in fact, the symmetrical flow of Fig. 7 is quite familiar from the BFM studies. A different behaviour of the lateral friction model is apparently confined to the intermediate range of $R$. We will concentrate on the flow in this parameter range in the next section where we will consider the transition of the lateral friction case to a model dominated by bottom friction. 

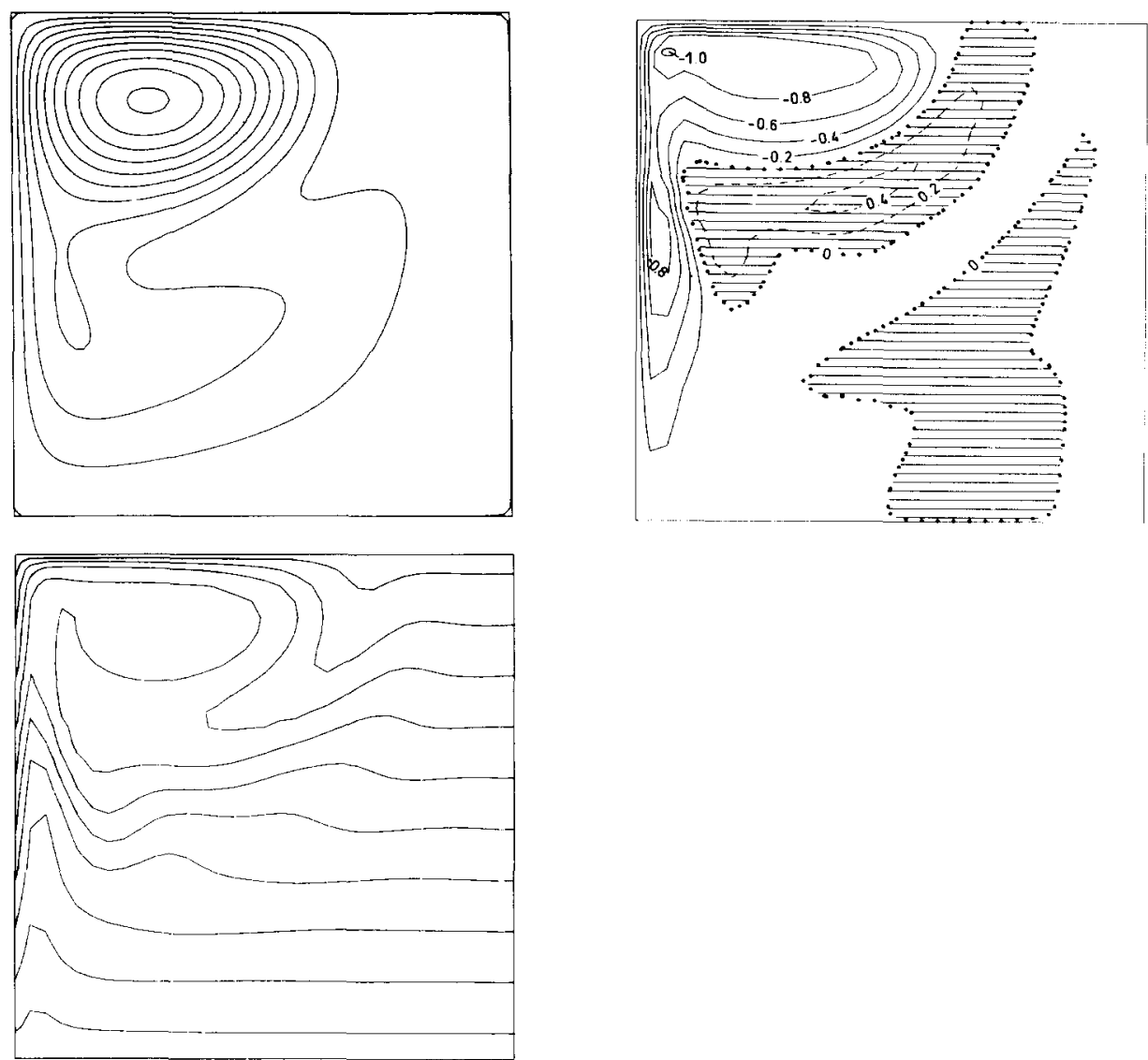

Fig. 6. $\psi, \zeta, q$ for experiment $5, \delta_{\mathrm{I}} / \delta_{\mathrm{L}}=1.0 . \psi_{\max }=8.92,\left|\zeta_{\max }\right|=101.3$. Contour intervals as in Fig. 3.

\section{TABLE II}

Experiments with lateral and bottom friction

\begin{tabular}{lllllll}
\hline Experiment & $E_{\mathrm{L}}$ & $E_{\mathrm{B}}$ & $R$ & $\delta_{\mathrm{I}} / \delta_{\mathrm{E}}$ & $\delta_{\mathrm{L}} / \delta_{\mathrm{B}}$ & Transport \\
\hline 5 & $9 \cdot 10^{-4}$ & - & $9.31 \cdot 10^{-3}$ & 1.0 & - & 89.2 \\
7 & $9 \cdot 10^{-4}$ & $9.7 \cdot 10^{-3}$ & $9.31 \cdot 10^{-3}$ & 1.0 & 10 & 53.7 \\
8 & $9 \cdot 10^{-4}$ & $2.4 \cdot 10^{-2}$ & $9.31 \cdot 10^{-3}$ & 1.0 & 4 & 37.8 \\
9 & $9 \cdot 10^{-4}$ & $4.8 \cdot 10^{-2}$ & $9.31 \cdot 10^{-3}$ & 1.0 & 2 & 26.7 \\
10 & 0 & $1.0 \cdot 10^{-1}$ & $1.00 \cdot 10^{-2}$ & 1.0 & 0 & 22.0 \\
11 & 0 & $1.0 \cdot 10^{-1}$ & $0.50 \cdot 10^{-2}$ & 2.0 & 0 & 25.7 \\
\hline
\end{tabular}

$\delta_{\mathrm{E}}$ is the larger of the two viscous scales, $\delta_{\mathrm{E}}=\max \left\{\delta_{\mathrm{L}}, \delta_{\mathrm{B}}\right\}$. 


\subsection{Transition within lateral and bottom friction}

Table II presents a sequence of experiments with a varying ratio of the lateral to the bottom friction scale $\delta_{\mathrm{L}} / \delta_{\mathrm{B}}$. We focus on the parameter range of oceanic interest, which yields a well-established NBC which does not pass across the whole basin, and take the value 1 for the ratio of the inertial to the (dominant) viscous scale $\delta_{\mathrm{E}}=\max \left\{\delta_{\mathrm{L}}, \delta_{\mathrm{B}}\right\}$. Figures 6, 8-11 show the transition from the lateral friction case to the bottom friction dominated one. The striking feature of the sequence is the gradual diminution of the strong sub-gyre coupled with a subsequent reduction of the maximum transport. Simultaneously, the region of negative vorticity becomes smaller, whereas $\left|\zeta_{\max }\right|$ in the northern corner is strong enough in all the flows to offset the
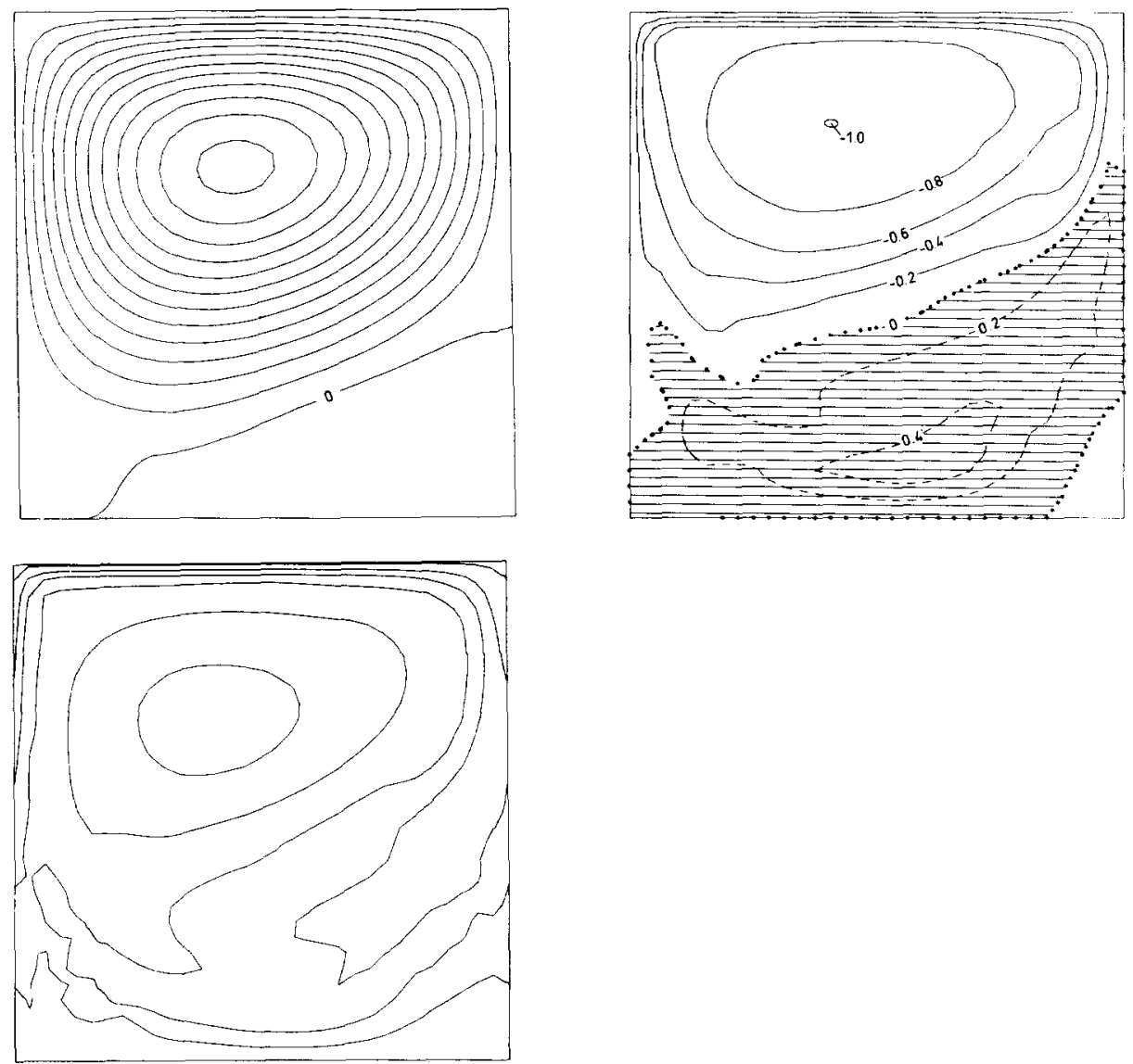

Fig. 7. $\psi, \zeta, q$ for experiment $6, \delta_{\mathrm{I}} / \delta_{\mathrm{L}}=1.5$ after 200 days of integration; $\psi_{\max }=42.5$, $\psi_{\min }=-3.38,\left|\zeta_{\max }\right|=126.2$. Contour intervals have been changed to $\Delta \psi=2.73, \Delta q=0.8$. $10^{-5} \mathrm{~s}^{-1}$. 

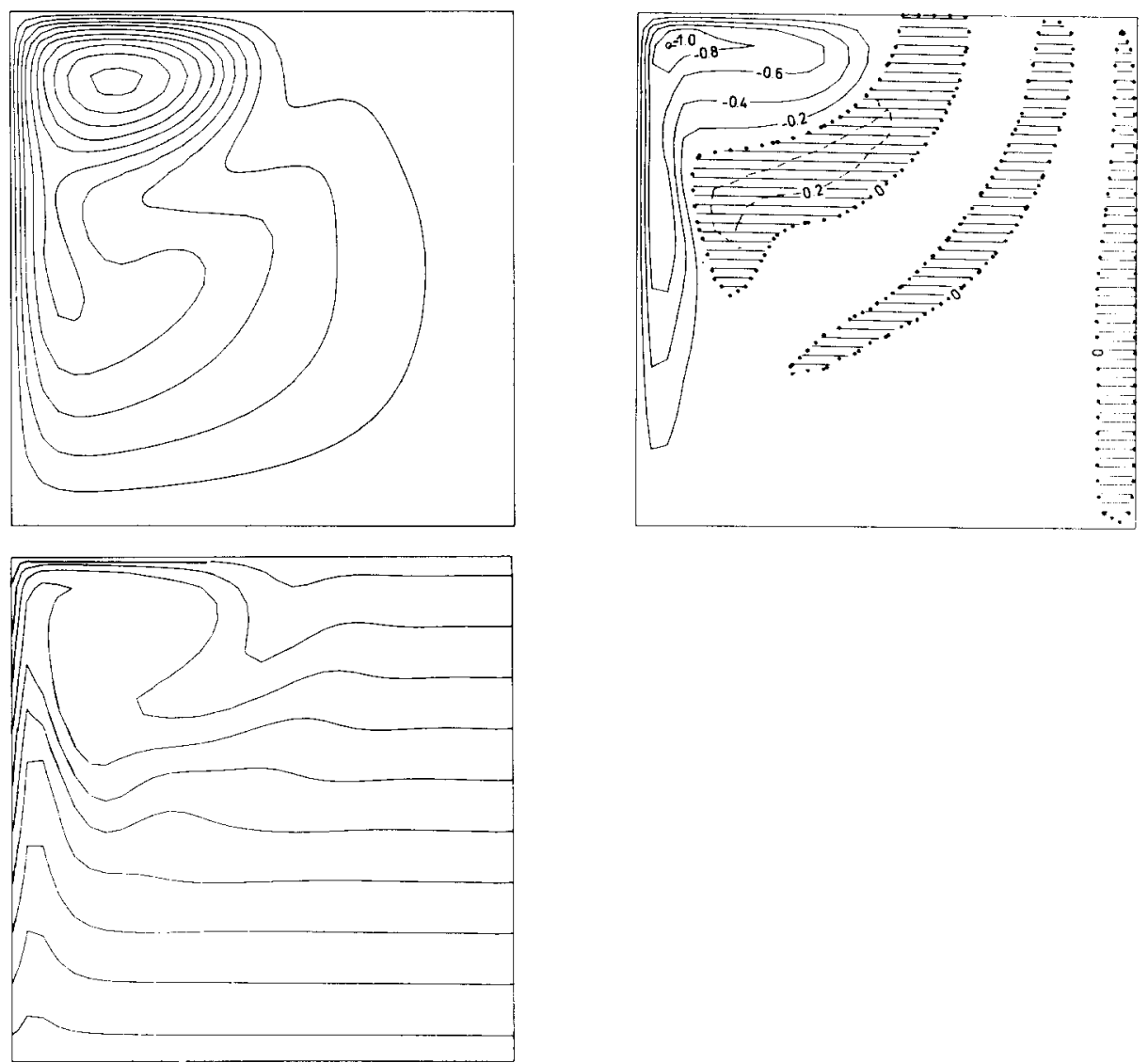

Fig. 8. $\psi, \zeta, q$ for experiment $7, \delta_{\mathrm{L}} / \delta_{\mathrm{B}}=10 ; \psi_{\max }=5.37,\left|\zeta_{\max }\right|=105.6 ; \Delta \psi=0.52$, the other contour intervals as in Fig. 3.

planetary vorticity gradient and to strongly deform the geostrophic contours. However, closed streamlines in the region of high $\zeta$ entirely disappear when bottom friction dominates.

However, a secondary effect in the sequence is that the penetration scale of the eastward jet becomes smaller suggesting that bottom friction is more effective to get rid of the vorticity in the western boundary layer. Another example for the effectiveness of the dissipation mechanism is that the NBC reaches the eastern wall in the lateral friction case for $\delta_{\mathrm{I}} / \delta_{\mathrm{L}} \sim 1.5$, whereas in the bottom friction model (Fig. 1) the inertial scale must be three or four times larger than the viscous scale. Thus the use of the ratio of the inertial scale to the larger of the two frictional scales seems inappropriate to examine the difference between the lateral friction and bottom friction case with respect to the recirculation behaviour. The contribution of both the dissipa- 

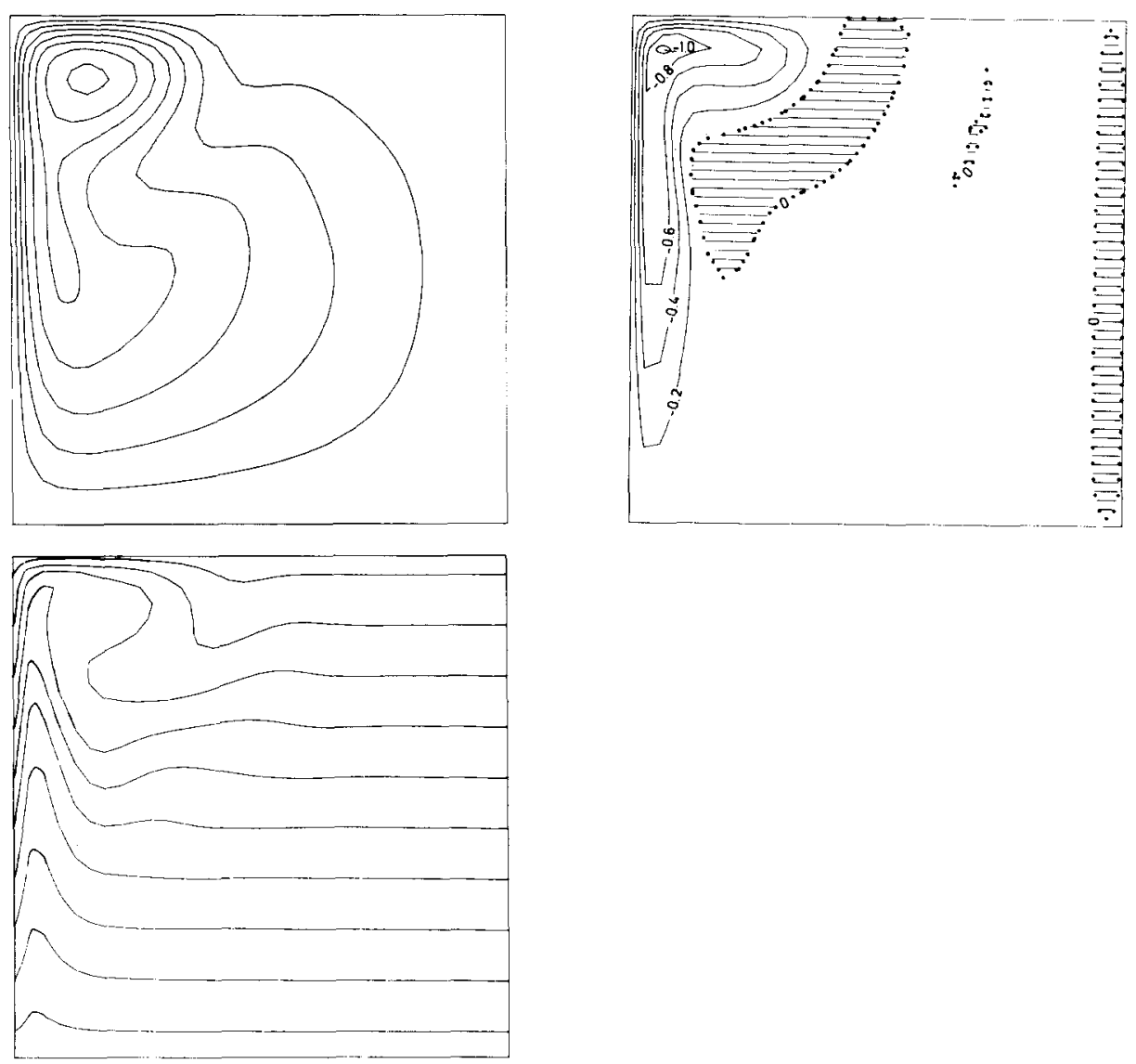

Fig. 9. $\psi, \zeta, q$ for experiment $8, \delta_{\mathrm{L}} / \delta_{\mathrm{B}}=4 ; \psi_{\max }=3.78,\left|\zeta_{\max }\right|=97.8$. Contour intervals as in Fig. 8 .

tion mechanisms in the experiments of this sequence is shown in Table III. The dissipation of energy is evaluated in multiplying the friction terms in the vorticity equation by $\psi$ and integrating over the area. Although the bottom friction scale in experiment 7 is only $1 / 10$ of the lateral friction scale, nearly $1 / 3$ of the energy dissipation is provided by bottom friction. The frictional mechanisms are equally important in experiment 8 , where $\delta_{\mathrm{L}}=4 \delta_{\mathrm{B}}$.

A bottom friction case, with a NBC penetrating about half the basin is obtained for $\delta_{\mathrm{I}} / \delta_{\mathrm{B}}=2.0$ (experiment 11, Fig. 12). This case is comparable to experiment 5 (Fig. 6) and helps to separate the specific effect of bottom friction from the effect due to more friction in general. A comparison of the flow fields in these two experiments very clearly shows the difference in the recirculation characteristics of the lateral and the bottom friction case. 

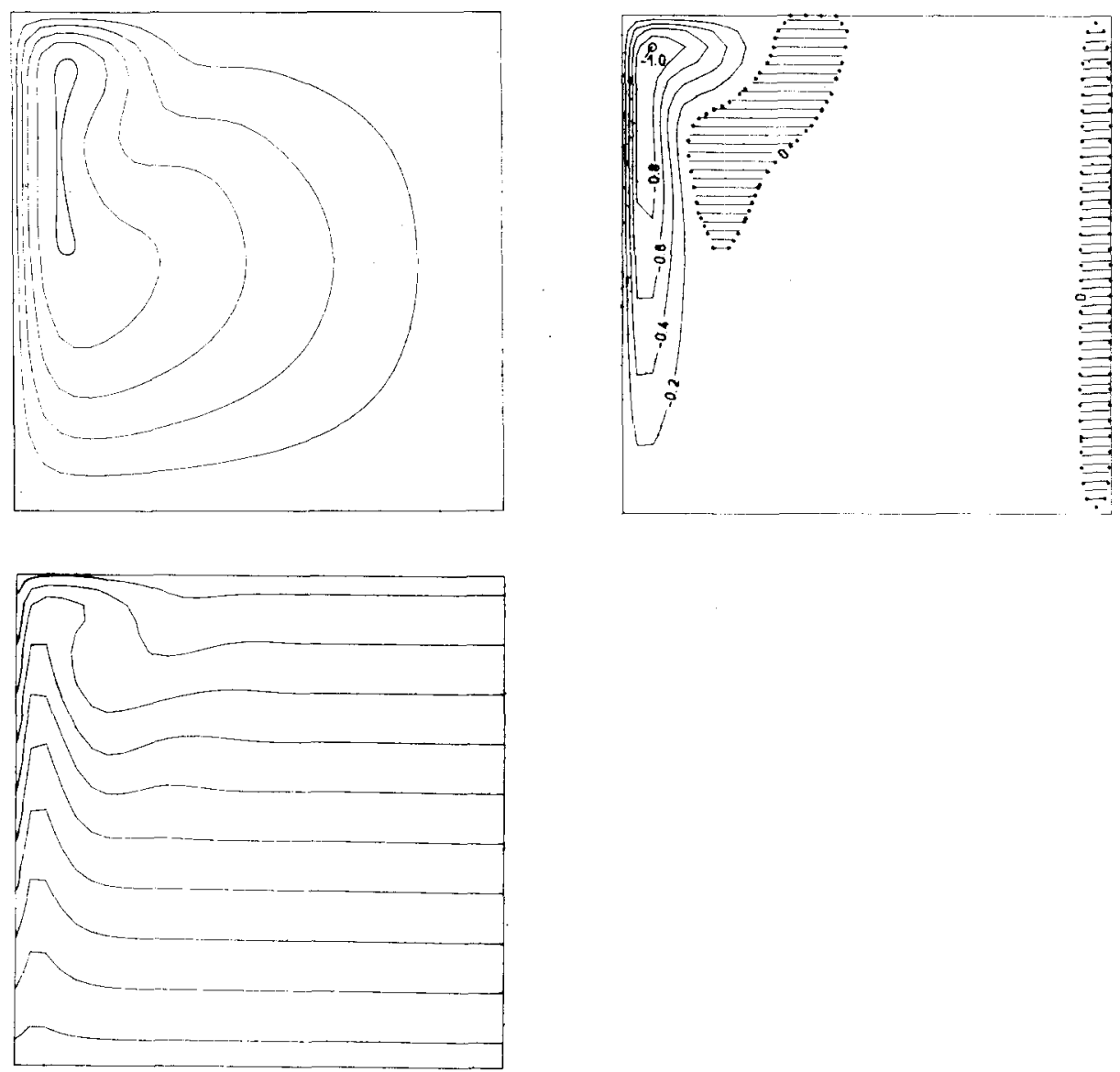

Fig. 10. $\psi, \zeta, q$ for experiment $9, \delta_{\mathrm{L}} / \delta_{\mathrm{B}}=2 ; \psi_{\max }=2.67,\left|\zeta_{\max }\right|=77.7$. Contour intervals as in Fig. 8 .

TABLE III

Percentage contribution to total, basin integrated dissipation by the two frictional mechanisms

\begin{tabular}{rccc}
\hline Experiment & $\begin{array}{l}\text { Frictional } \\
\text { mechanism } \\
\delta_{\mathrm{L}} / \delta_{\mathrm{B}}\end{array}$ & $\begin{array}{l}\text { Bottom friction } \\
E_{\mathrm{B}} \int \psi \nabla^{2} \psi \mathrm{d} x \mathrm{~d} y\end{array}$ & $\begin{array}{l}\text { Lateral friction } \\
E_{\mathrm{L}} \int \psi \nabla^{4} \psi \mathrm{d} x \mathrm{~d} y\end{array}$ \\
\hline 5 & - & 0 & 100 \\
7 & 10 & 29.2 & 70.8 \\
8 & 4 & 47.2 & 52.8 \\
9 & 2 & 65.0 & 35.0 \\
10 & 0 & 100 & 0 \\
\hline
\end{tabular}



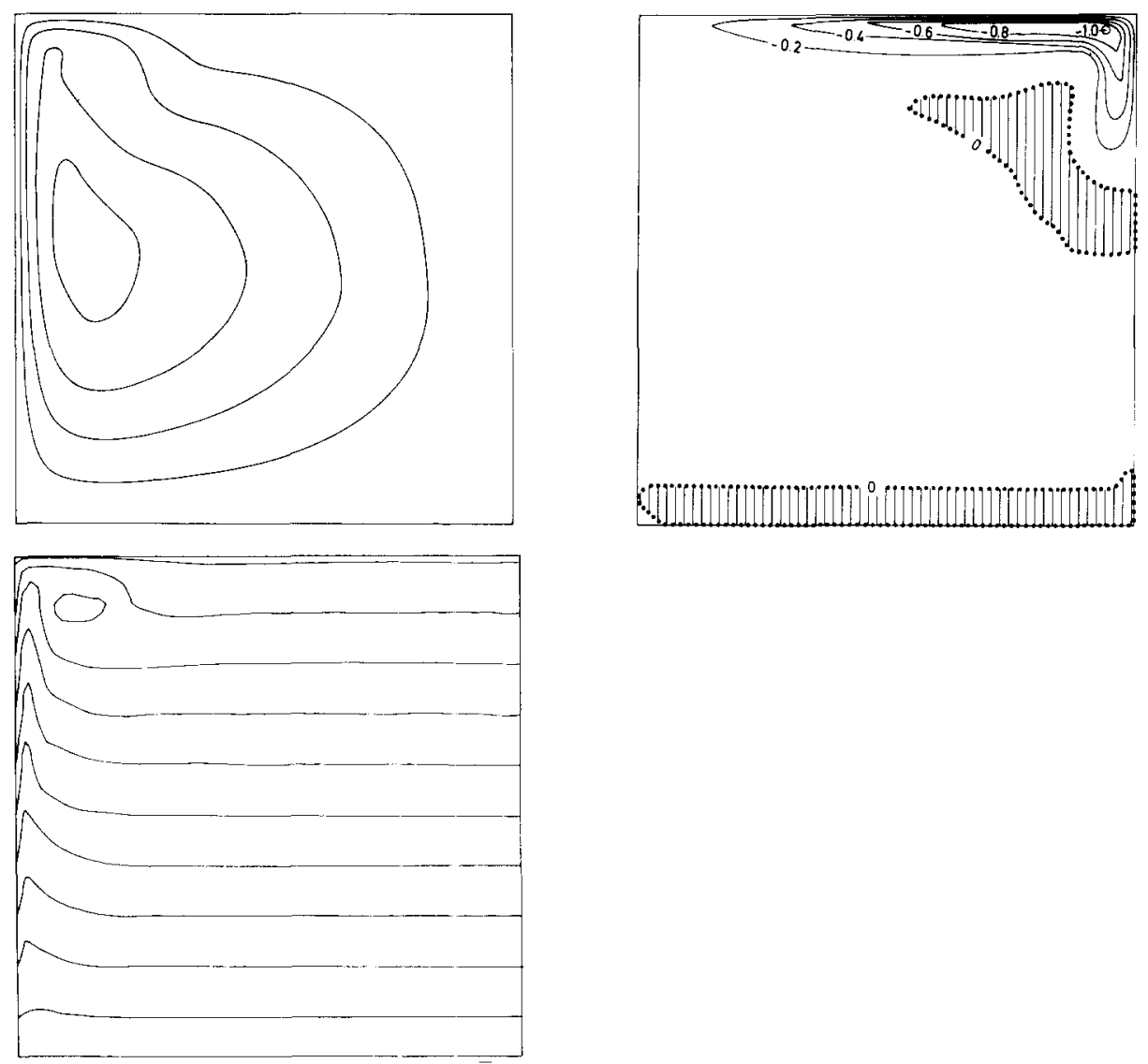

Fig. 11. $\psi, \zeta, q$ for experiment $10, \delta_{\mathrm{L}} / \delta_{\mathrm{B}}=0, \delta_{\mathrm{I}} / \delta_{\mathrm{B}}=1 ; \psi_{\max }=2.20,\left|\zeta_{\max }\right|=79.0$. Contour intervals as in Fig. 8.

It has to be noted that all computations in this paper were performed with the same (free-slip) boundary conditions to separate the effects of the boundary conditions and the frictional parameterization. In the bottom friction case we have to add an-arbitrarily small-amount of lateral friction to make the free-slip condition mathematically consistent. This implies that the bottom friction dominated case is not quite the same as Veronis' BFM without any dynamical boundary conditions. However, as long as $\delta_{\mathrm{B}}>\delta_{\mathrm{L}}$, only slight differences have shown up between the BFM solutions and the free-slip model due to the different boundary layer structures. A similar result has been found by Blandford (1971). We omit a presentation of a bottom friction/free-slip sequence with varying $R$ because it gives no essentially new information compared to the BFM. 

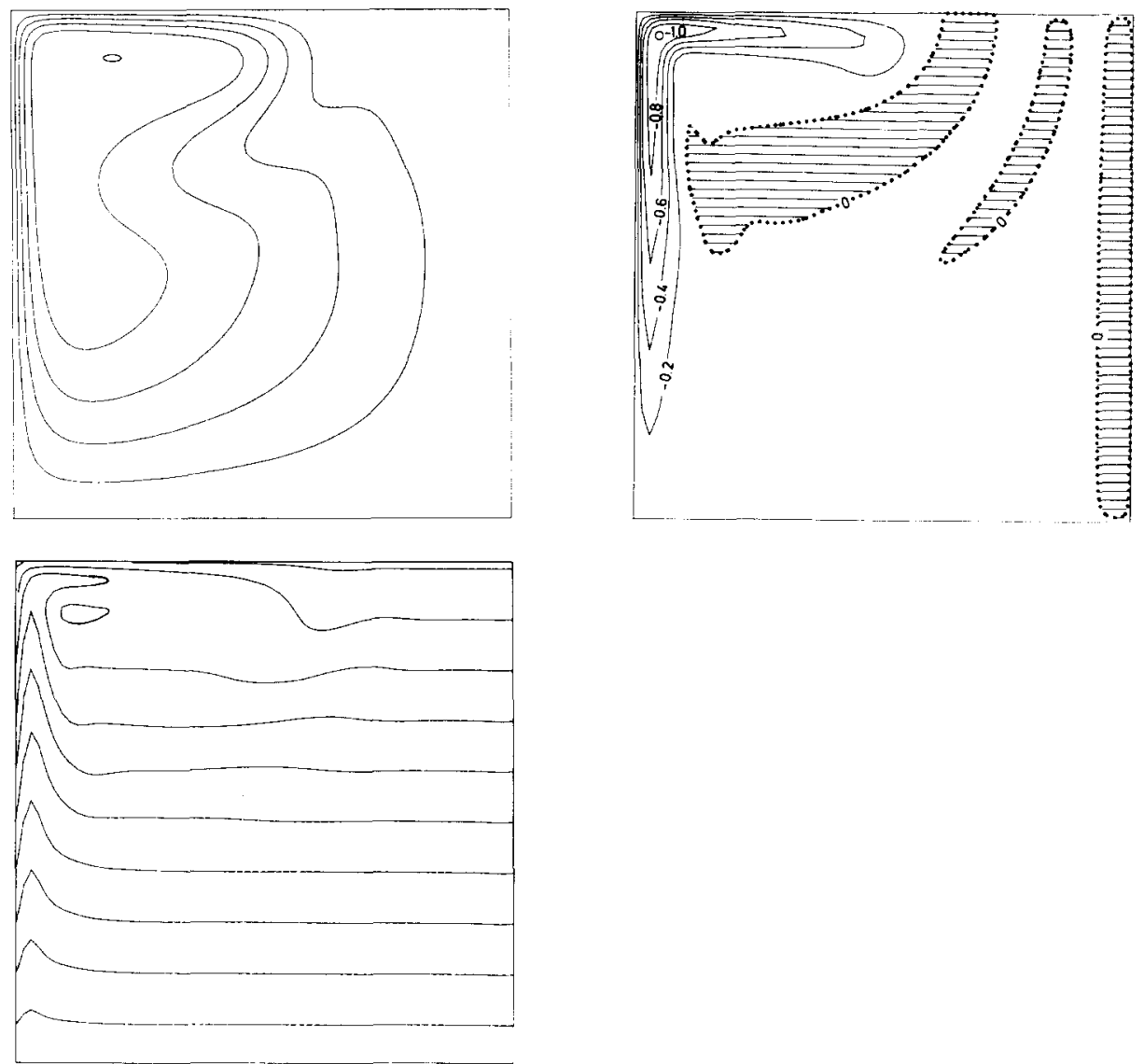

Fig. 12. $\psi, \zeta, q$ for experiment $11, \delta_{\mathrm{L}} / \delta_{\mathrm{B}}=0, \delta_{\mathrm{I}} / \delta_{\mathrm{B}}=2 ; \psi_{\max }=2.57,\left|\zeta_{\max }\right|=143.0$. Contour intervals as in Fig. 8.

\section{TABLE IV}

Experiments with decreasing viscous scale. Transport values are given for the anticyclonic gyre in these double-gyre forced experiments

\begin{tabular}{llllll}
\hline $\begin{array}{l}\text { Experi- } \\
\text { ment }\end{array}$ & $E_{\mathrm{L}}$ & $R$ & $\delta_{\mathrm{I}} / \delta_{\mathrm{L}}$ & $\begin{array}{l}\text { Transport } \\
\left(10^{12} \mathrm{~cm}^{3} \mathrm{~s}^{-1}\right)\end{array}$ & $\begin{array}{l}\text { Solution } \\
\text { behaviour }\end{array}$ \\
\hline 12 & $2.4 \cdot 10^{-3}$ & $1.1 \cdot 10^{-2}$ & 0.8 & 44.2 & steady \\
13 & $6.0 \cdot 10^{-4}$ & $4.6 \cdot 10^{-3}$ & 0.8 & 65.0 & steady \\
14 & $1.0 \cdot 10^{-4}$ & $1.4 \cdot 10^{-3}$ & 0.8 & 87.4 & quasi-steady \\
15 & $4.2 \cdot 10^{-5}$ & $7.8 \cdot 10^{-4}$ & 0.8 & 92.3 & quasi-steady \\
16 & $5.0 \cdot 10^{-5}$ & $1.1 \cdot 10^{-3}$ & 0.9 & 105.8 & quasi-steady
\end{tabular}

The solutions are antisymmetric with respect to mid-latitude and the total transport of the eastward jet is twice this value. 


\subsection{The range of steady solutions}

As dictated by the finite resolution, the diffusivities used in the calculations are still somewhat higher than the values to be expected upon consideration of the oceanic scales in section 2 . We are left with the question whether the character of the solutions would remain the same if the imposed boundary layer scales were allowed to be smaller and, thus, more closely related to oceanic values.

Table IV lists a sequence of lateral friction experiments with decreasing values of $E_{\mathrm{L}}$ and $R$. The ratio $\delta_{\mathrm{I}} / \delta_{\mathrm{L}}$ is held constant at the value 0.8 in the experiments $12-15$, and is 0.9 in experiment 16 . These calculations were made by means of double-gyre wind forcing $\left(\operatorname{curl}_{z} \tau=-\sin 2 y\right)$. They are part of an on-going study on mesoscale resolution (eddy resolving) barotropic circulation models. For the purposes of the present paper, however, the particular forcing is not relevant and we may focus attention on the transport features of the anticyclonic gyre only.

Approaches to equilibrium for some experiments are shown in Fig. 13 where the kinetic energy is plotted against time. In the sequence a steady state is reached for the experiments with $E_{\mathrm{L}} \geqslant 6 \cdot 10^{-4}$ (equivalent to an eddy coefficient $A_{\mathrm{H}}=3.1 \cdot 10^{7} \mathrm{~cm}^{2} \mathrm{~s}^{-1}$ in the $2000 \mathrm{~km}$ basin). Decreasing of the viscous scale results in a much larger spin-up time: it grows from 150 days for $E_{\mathrm{L}}=2.4 \cdot 10^{-3}\left(A_{\mathrm{H}}=1.2 \cdot 10^{8} \mathrm{~cm}^{2} \mathrm{~s}^{-1}\right)$ to around 400 days for $E_{\mathrm{L}}=6 \cdot 10^{-4}$. This may be explained by the relation $\zeta_{\mathrm{t}}=E_{\mathrm{L}} \nabla^{2} \zeta$ which implies that the decay times for initial oscillations become longer as the diffusivity becomes smaller.

The situation changes fundamentally with respect to $E_{\mathrm{L}}=1 \cdot 10^{-4}\left(A_{\mathrm{H}}=\right.$ $5.2 \cdot 10^{6}$ ) where a steady solution is no longer obtained. After an initial spin-up time of 600 or 700 days fluctuations arise, settling down in a statistically steady state with regular oscillations. Figure 14 gives the time
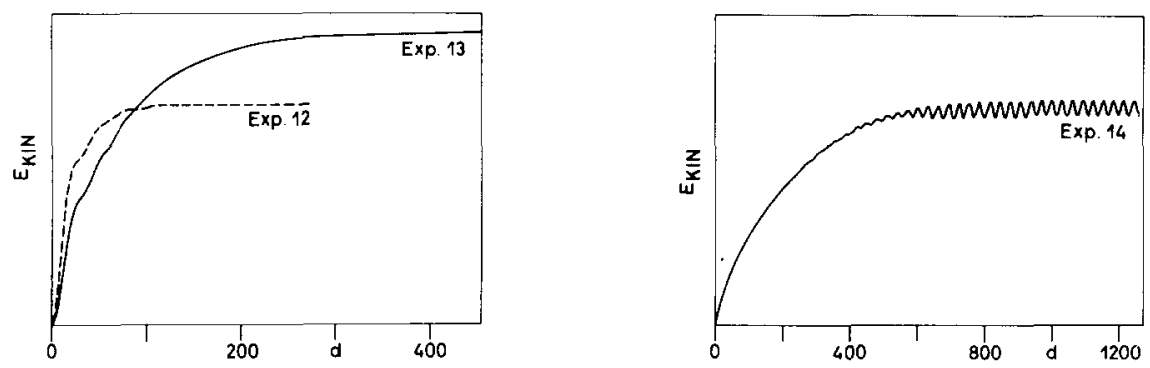

Fig. 13. Basin integrated kinetic energy (in arbitrary units) against time for experiments 12,13 and 14. 

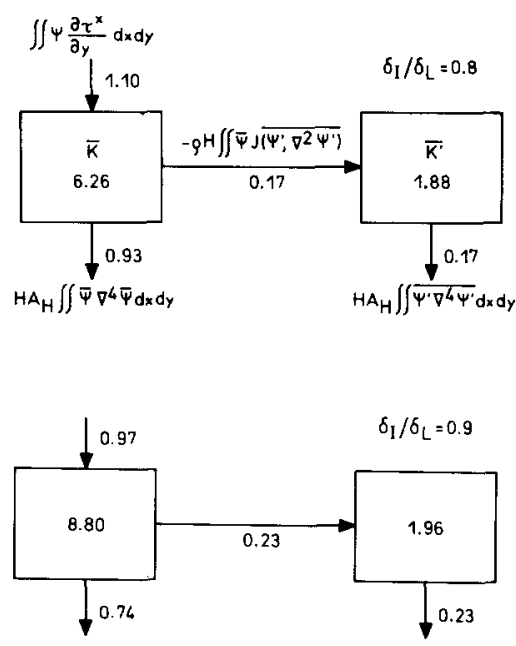

Fig. 14. Energy box diagrams showing the kinetic energy of the mean field $(K)$, the mean kinetic energy of the fluctuations $\left(K^{\prime}\right.$ ) (in $10^{6} \mathrm{erg} \mathrm{cm}^{2}$ ) and the energy transfer rates (in erg $\left.\mathrm{cm}^{2} \mathrm{~s}^{-1}\right)$ for experiments 14 and 16 .

mean energetics in the quasisteady states of experiments 14 and 16. The friction acting on the mean state is not able to dissipate the total wind input of energy. The mean states are balanced by a transfer of energy to the fluctuating part of the solutions by Reynolds stress interaction work. The values indicate that the significance of the eddies in the solutions increases with increasing non-linearity: for $\delta_{\mathrm{I}} / \delta_{\mathrm{L}}=0.8,15 \%$ of the wind energy goes into the eddy field, whereas for $\delta_{1} / \delta_{\mathrm{L}}=0.9$ it is $23 \%$.

We will not go into details of the quasi-steady solutions here. The properties of the fluctuating component in these experiments and the dynamical interactions between the mean flow and the eddy field shall be discussed in a separate paper. In the present study our attention is restricted to a particular aspect of the time mean part of the flow: namely, the recirculation character of the steady lateral friction model is not qualitatively altered when the range of the steady solutions is left and interaction with eddies becomes an integral part of the flow.

Figure 15 and 16 display the equilibrium streamfunction contours of the experiments 12 and 13, Figs. 17 and 18 show the time mean parts of the quasi-steady solutions of experiments 14 and 15 . The sequence, based on a fixed $\delta_{\mathrm{I}} / \delta_{\mathrm{L}}=0.8$, shows qualitative similar flow patterns and, thus, confirms the relevance of the $\delta_{1} / \delta_{\mathrm{L}}$-ratio as a suitable measure of non-linearity. When decreasing the scales $\delta_{\mathrm{I}}, \delta_{\mathrm{L}}$, the NBC becomes narrower and the basinintegrated transport stronger. It is important to note that this enhancement of the sub-gyre circulation takes place even in the range of the steady 


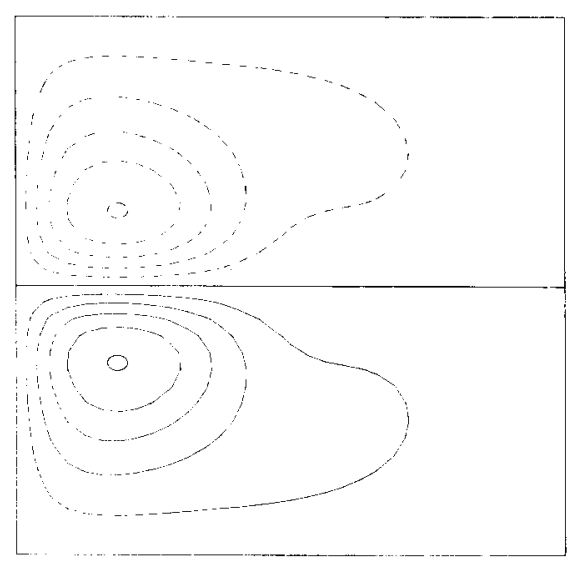

Fig. 15. Streamfunction $\psi$ in the steady state of experiment $12 ; \psi_{\max }=4.42, \Delta \psi=0.89$.

solutions. It is not obvious whether the eddy field additionally contributes to the recirculation strength in any way, because the two effects, i.e., the reduction of $E_{\mathrm{L}}$ and the existence of fluctuations, cannot be separated as one moves into the quasi-steady regime. The similarity of the recirculation region in both the steady and the quasi-steady solutions, however, indicates a minor influence of the eddies on the mean transport field.

Another feature of the sequence-similar to EGCM stream-function fields-is the eastward bending of the streamlines outside the recirculation region in Figs. 17 and 18. A secondary eastward current emerges south of the sub-gyre when the range of steady solutions is left. The question whether this feature is caused by the dynamical influence of the eddy field will be dealt with in detail in the forthcoming paper.

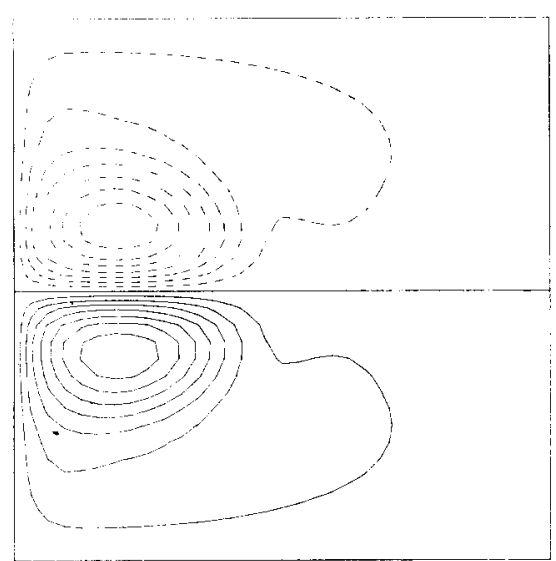

Fig. 16. Streamfunction $\psi$ in the steady state of experiment $13 ; \psi_{\max }=6.50, \Delta \psi=0.89$. 


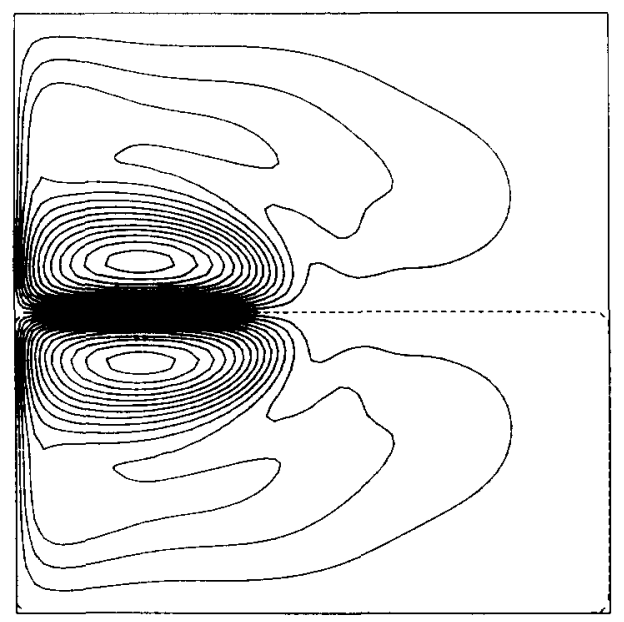

Fig. 17. Mean streamfunction $\bar{\psi}$ in the quasi-steady state of experiment $14 ; \bar{\psi}_{\max }=8.74$, $\Delta \bar{\psi}=0.60$.

We may summarize this section as follows: contrary to the bottom friction model, in the lateral friction/free-slip model a strong recirculating sub-gyre emerges which increases the basin transport several times. This feature is part of both the steady and quasi-steady solutions which are obtained by decreasing the model diffusivity.

In the next section we will discuss the dynamics in the recirculation region.

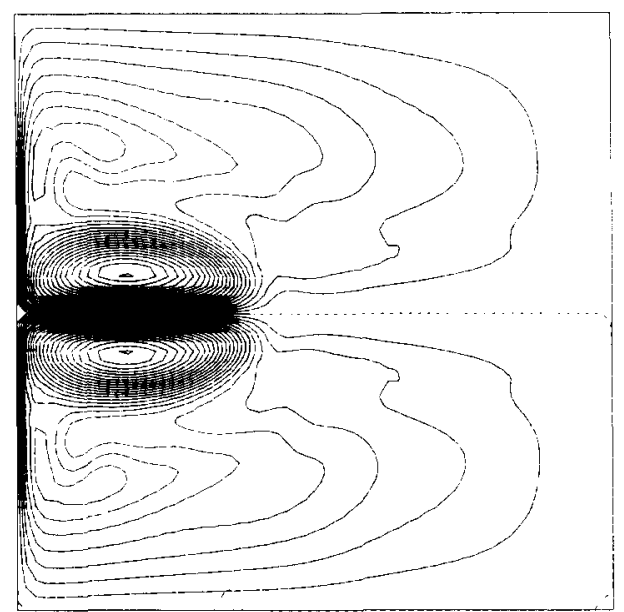

Fig. 18. Mean streamfunction $\bar{\psi}$ in the quasi-steady state of experiment $15 ; \bar{\psi}_{\max }=9.23$. $\Delta \bar{\psi}=0.40$. 


\section{DISCUSSION OF THE VORTICITY DYNAMICS}

\subsection{The integral vorticity constraint}

We want to understand the different structures of the steady solutions with bottom and lateral friction. The type of friction influences the solution of the BVE most strongly in the parameter range of 'intermediate' non-linearity. Let us compare, for example, the results of experiment 5 (Fig. 6) and experiment 11 (Fig. 12) which exhibit almost the same NBC penetration scale. In the case of lateral diffusion there are closed streamlines within the region of negative vorticity, i.e., in the region of closed or almost closed isostrophes. In the bottom friction case the region of negative vorticity is limited to the boundary layer and, thus, all streamlines close in a region where $|\zeta|$ is small. Accordingly, we get a strong, tight recirculation pattern in the first, and a smooth return flow in the latter case.

We base our arguments for the different recirculation intensities in the near field on the integral balance of vorticity for a column in one complete circuit. Taking the closed line integral of the BVE (2.2) along any streamline $\psi=$ constant, the vorticity transport terms drop out and

$\oint \tau \cdot \mathrm{d} s=E_{\mathrm{B}} \phi \boldsymbol{u} \cdot \mathrm{d} \boldsymbol{s}-E_{\mathrm{L}} \oint \nabla \zeta \cdot \mathrm{d} \boldsymbol{n}$

remains in the steady state, or equivalently

$$
\iint_{A} \operatorname{curl}_{z} \tau \mathrm{d} x \mathrm{~d} y=E_{\mathrm{B}} \iint_{A} \zeta \mathrm{d} x \mathrm{~d} y-E_{\mathrm{L}} \iint_{A} \nabla^{2} \zeta \mathrm{d} x \mathrm{~d} y
$$

The circuit is taken counterclockwise; $\boldsymbol{n}$ is the outer normal to $\boldsymbol{s}$. In equilibrium the vorticity input from the wind $(W)$ into any domain $\mathrm{A}$, enclosed by a streamline, must be balanced by the model dissipation process. This relation holds whatever the local vorticity balances, i.e., whatever the local dynamics, along the circuit may be.

Let us consider the model with bottom friction alone. With $Z$ as the mean magnitude of $\zeta$ in $\mathrm{A}$, we get $W \sim E_{\mathrm{B}} Z$. For a given windstress and frictional parameter, the amount of vorticity enclosed by a streamline solely depends on the area the streamline encompasses: for a small area the wind input of vorticity $W$ is small. Consider a closed circuit confined to the northwestern area corresponding to, say, $W=0.5$ in the model with $E_{\mathrm{B}}=5 \cdot 10^{-2}$. Therefore, $Z \sim W / E_{\mathrm{B}}=0(10)$. As the vorticity in the boundary layer, in a non-linear case like experiment 11 , is an order of magnitude larger, the column must pass a more quiet region somewhere on its way to keep the average vorticity small.

We see that the linear friction law prevents the flow from a recirculation in a small region of large $|\zeta|$, because the wind-input of vorticity cannot 


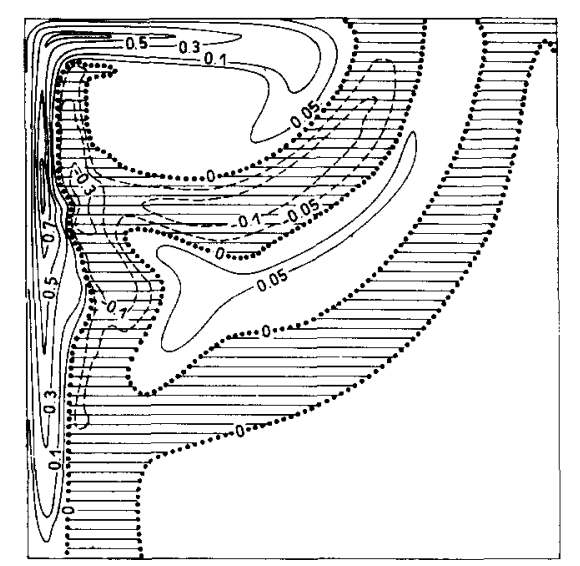

Fig. 19. Contour map of $\nabla^{2} \xi$, normalized with respect to its maximum value, for experiment 5. Regions of negative diffusion are shaded. The isolines shown are $\pm 0.05, \pm 0.1$, $\pm 0.3, \ldots, 0.9\left|\nabla^{2} \zeta_{\max }\right|$.

account for the large vorticity loss which would occur along such a trajectory. Thus, this particular form of model friction puts a strong constraint on the possible type of recirculation, especially in the non-linear cases with strong inertial boundary layers.

On the other hand, if such large values of $R$ are considered which yield an inertial recirculation - the modified Fofonoff-type of circulation (see, for example, Veronis, 1966b) - the flow must lose its boundary layer character, i.e., the vorticity will spread out more uniformly over the basin. With $U$ and $\lambda$ as the average current speed and length scale, we may assume $Z \sim U / \lambda$. Increasing the speed of a particle on its circuit demands $\lambda \propto U$; this is the same relation which Harrison and Stalos (1982) found by their scale analysis for the NBC in the bottom friction experiment.

In the intermediate range of $R$ the situation changes if the vorticity loss is modelled by a higher order friction law. Clearly, a strong recirculation with large $|\zeta|$ can occur, provided $\nabla^{2} \zeta$ remains small. This is what happens in the experiments. Figure 19 shows the distribution of $\nabla^{2} \zeta$ for experiment 5 . Dissipation, i.e., diffusion of negative vorticity out of the area bounded by a streamline, occurs all along the boundary jets in the west and north but is small in the tight recirculation zone. Outside the sub-gyre area the term takes the opposite sign. Negative vorticity is diffused inwards here and accelerates the circulation.

\subsection{Regional vorticity balances}

An integral vorticity balance along a complete circuit gives no insight into the physical processes acting on a fluid column. Locally the vorticity 
transport terms, i.e., advection of relative and planetary vorticity, may be the important terms and dominate the vorticity balances. We concentrate on the dynamics of the sub-gyre in the lateral diffusion model (experiments 4 and $5)$. Denote the terms in the vorticity eq. 2.2 by

$N=R(\boldsymbol{u} \cdot \nabla \zeta) \quad B=v \quad W=\operatorname{curl}_{z} \tau \quad F=-E_{\mathbf{L}} \nabla^{2} \zeta$

thus

$N+B+W+F=0$

The strong recirculation takes place almost entirely in the region of $\zeta<0$ or, equivalently, in the region of the closed geostrophic contours. Thus, a column in the sub-gyre will not change its potential vorticity very much. Suppose, in a first approximation, $\mathrm{d} q / \mathrm{d} t \approx 0$; from this we expect local balances between advection $N$ and beta $B$ and, simultaneously, between wind $W$ and friction $F: N \sim B, W \sim F$. In a regional vorticity budget this will hold provided there is no cancellation of the locally dominant terms. We consider the eastern part of the sub-gyre with southward flow to compare the budgets of the steady lateral friction model with the depth integrated vorticity budget of the EGCM - sub-gyre analysed by Harrison and Holland (1981). This is their region ' $R E C I R C$ '.

Table $\mathrm{V}$ shows the general tendency of the budgets in the steady lateral friction model and in the EGCM towards the expected behaviour of a free inertial recirculation. In the EGCM the advection term contains both the advection of vorticity by the mean flow $(M A D=166)$ and the mean advection by the eddies $(E A D=-7)$. Although this is a case with strong eddy activity, the basic balance in the sub-gyre recirculation is between mean advection and beta; the (lateral) eddy flux-divergence is rather unimportant compared with the mean advection.

\subsection{Dynamical regimes in the lateral friction model}

The aim of a vorticity analysis is to identify the dominant physical processes in the various parts of the gyre. Here we follow the approach of

TABLE V

Vorticity budgets in the recirculation region (RECIRC)

\begin{tabular}{llrll}
\hline & \multicolumn{1}{c}{$B$} & $N$ & $W$ & $F$ \\
\hline Experiment 4 & -85 & 94 & 11 & -21 \\
Experiment 5 & -195 & 199 & 39 & -45 \\
EGCM & -166 & 159 & 22 & -16 \\
\hline
\end{tabular}

The EGCM values are basin depth integrals $\left(\times 10^{6} \mathrm{~cm}^{3} \mathrm{~s}^{-2}\right)$ from Harrison and Holland (1981); the values of the experiments 4 and 5 are in dimensionless units. (The values are only internally consistent for each experiment and cannot directly be compared.) 


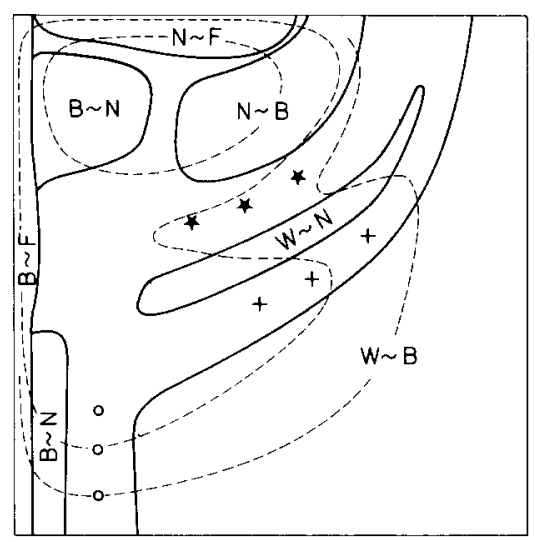

Fig. 20. Dynamic regimes of the flow (experiment 5). See text. In the transition regions the types of balance are:

$* * * W+F \sim B$

$+++W \sim B+F$

$\circ \circ \circ W+F \sim N$

Veronis (1966b) to define characteristic regimes of the flow, each representing a region of a specific, dynamical balance of forces. We consider the percentage contribution of each term in the vorticity equation at every grid point. Let us denote these contributions, again, as $N, B, W, F$. Because of the variety of types of balance between the four terms in the basin, it seem most appropriate to firstly seek those regions where the dynamics are dominated by two terms only.

Figure 20 shows the regimes of experiment 5 , which are characterized by balances between two terms which together contribute more than about $80 \%$ to the balance in the equation $N+B+W+F=0$. Thus, these terms are of opposite sign and nearly balance each other. The terms on the left hand side of the relations are of the same sign as the wind-stress curl.

The Sverdrup regime $(W \sim B)$ is seen to fill the entire southeast half of the basin. Columns entering the western boundary layer first pass a regime where the inertial terms dominate. Friction is significant along the western and northern boundary. Near the western boundary a Munk-layer balance holds, whereas the eastward flowing jet is of advective, diffusive character. The recirculation region is characterized by free advective dynamics.

\section{CONCLUSIONS}

The wind-driven ocean circulation problem is studied by numerical integrations of the barotropic vorticity equation (BVE), subject to steady zonal wind forcing. Parameterizing the vorticity sink by lateral diffusion of vorticity in connection with free-slip conditions at the boundaries leads to a new 
kind of solution of the BVE in the geophysically meaningful range of parameters (that is, of moderate non-linearity):

steady as well as quasi-steady solutions are possible, depending on the (prescribed) diffusivity of the flow;

in contrast to the bottom friction case (Veronis, 1966b; Harrison and Stalos, 1982) a strong recirculating sub-gyre emerges in the northwestern corner of the basin increasing the maximum transport of the northern boundary current several times above the Sverdrup transport. The shape as well as the vorticity dynamics of the recirculation seem to be very similar to the mean transport fields of recently investigated quasi-steady EGCMs; and

this intense sub-gyre is a feature of both the quasi-steady and the steady solutions of the BVE.

Altogether, the essential features of the EGCM-transport fields appear to be explainable in terms of a simple barotropic system where lateral friction represents the dominant dissipation mechanism. The possibility of reproducing these features in steady solutions lends strong support to Marshall's (1984) suggestion of the applicability of a down-gradient parameterization of the divergent eddy vorticity fluxes. His rationalization of the flux in two dynamically distinct contributions might explain Harrison's (1978) relative lack of success in establishing a local flux-gradient relationship for EGCMfields, an otherwise paradoxical result regarding the present study.

It is interesting to note that the steady lateral friction/free-slip solutions as described here are qualitatively very similar to the mean fields of the unstable no-slip solutions of Kamenkovich et al. (1985) with eddies generated in the western boundary layer. Different dynamical boundary conditions strongly determine the behaviour of the boundary currents, i.e., lead to instabilities in the western boundary layer (no-slip), avoid these instabilities

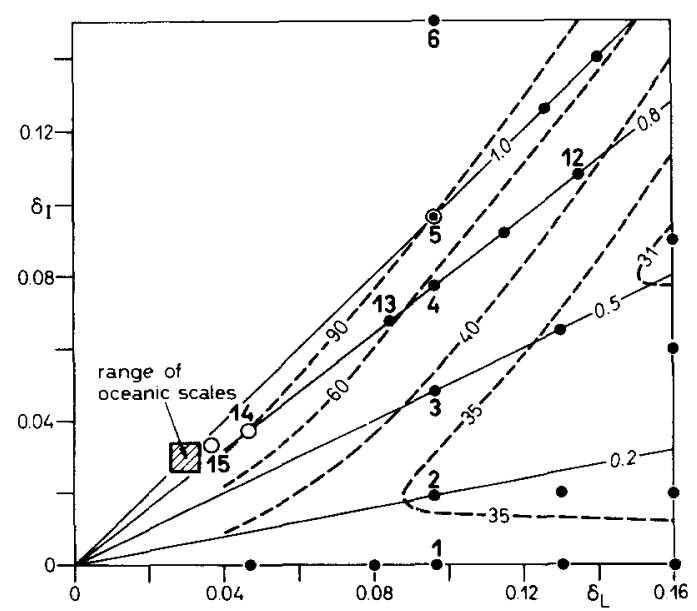

Fig. 21. Isolines of maximum transport (dashed lines) as a function of the model parameters, $\psi_{\max }\left(\delta_{1}, \delta_{L}\right)$, for the barotropic model with lateral friction/free-slip. 
and allow eastward jets (free-slip), or concentrate $q$ contours at the eastward jet causing vigorous instabilities (Marshall); but it seems they do not influence the recirculation behaviour of the interior flow.

The results of all the calculations made are summarized in Fig. 21 which displays the dependence of the maximum transport on the external parameters $\delta_{\mathrm{I}}$ and $\delta_{\mathrm{L}}$. Parameter combinations between the lines $\delta_{\mathrm{I}}=0.8 \delta_{\mathrm{L}}$ and $\delta_{1}=1.0 \delta_{\mathrm{L}}$ result in flow cases most like the ocean, in the sense that they exhibit a Sverdrup regime in the larger part of the basin, a western and northern boundary current with a transport increasing downstream and a penetration scale of one-third or one-half of the basin width, coupled with a tight recirculation regime. Approaching the small 'oceanic' boundary layer scales $\left(\delta_{\mathrm{I}}, \delta_{\mathrm{L}} \sim 1 / 100\right.$ basin width) along these lines we observe an increase of the maximum transport to values of about $100 \mathrm{~Sv}$ or more than three times the Sverdrup transport.

\section{ACKNOWLEDGEMENTS}

This paper is based on portions of the author's doctoral dissertation. I thank Prof. W. Krauss for his supervision of this work. Appreciation is also due to Prof. J. Willebrand, Drs. J. Kielmann, R.H. Käse, P.G. Hardtke and Chr. Wübber for helpful discussions and comments leading to improvements in the manuscript. This work was a contribution to the Sonderforschungsbereich 133 and was supported by the Deutsche Forschungsgemeinschaft.

\section{REFERENCES}

Arakawa, A., 1966. Computational design for long-term numerical integration of the equations of fluid motion: Two dimensional incompressible flow. Part I. J. Comput. Phys., 1: $119-143$.

Blandford, R.R., 1971. Boundary conditions in homogeneous ocean models. Deep-Sea Res., 18: $739-751$.

Böning, C., 1985. Eine Untersuchung der Dynamik der windgetriebenen ozeanischen Zirkulation mit einem wirbelauflösenden barotropen Modell. Ber. Inst. Meereskunde Kiel, No. 137, pp. 149.

Briggs, W.L., 1980. A new class of steady solutions of the barotropic vorticity equation. Dyn. Atmos. Oceans, 4: 67-99.

Bryan, K., 1963. A numerical investigation of a nonlinear model of a wind-driven ocean. $J$. Atmos. Sci., 20: 594-606.

Fofonoff, N.P., 1954. Steady flow in a frictionless homogeneous ocean. J. Mar. Res., 13: 254-262.

Gottlieb, D. and Orszag, S.A., 1977. Numerical analysis of spectral methods: Theory and applications. CBMS-NSF Monograph number 26, Society for Industrial and Applied Mathematics, Philadelphia, 172 pp.

Haidvogel, D.B., Robinson, A.R. and Schulman, E.E., 1980. The accuracy, efficiency, and stability of three numerical models with application to open ocean problems. J. Comput. Phys., 34: 1-53. 
Harrison, D.E., 1978. On the diffusion parameterization of mesoscale eddy effects from a numerical ocean experiment. J. Phys. Oceanogr., 8: 913-918.

Harrison, D.E., 1982. On deep mean flow generation mechanisms and the abyssal circulation of numerical model gyres. Dyn. Atmos. Oceans, 6: 135-152.

Harrison, D.E. and Holland, W.R., 1981. Regional eddy vorticity transport and the equilibrium vorticity budgets of numerical model ocean circulation. J. Phys. Oceanogr., 11: 190-208.

Harrison, D.E. and Stalos, S., 1982. On the wind-driven ocean circulation. J. Mar. Res., 40: $773-791$.

Holland, W.R., Harrison, D.E. and Semtner, A.J., Jr., 1983. Eddy-resolving numerical models of large-scale ocean circulation. In: A.R. Robinson (Editor), Eddies in Marine Science. Springer-Verlag, Berlin, 609 pp.

Kamenkovich, V.M., Belozerkovsky, S.O. and Panteleyer, M.S., 1985. K voprosu o chislennom modelirovany barotropych techeny, poroshdennych Krupnomasshtabnym polem vetra. Isvestiya Polymode, Vypusk 15, Moskva, 1985.

Krauss, W. and Wübber, C., 1982a. The response of the North Atlantic to annual wind variations along the eastern coast. Deep-Sea Res., 29: 851-868.

Krauss, W. and Wübber, C., 1982b. A semispectral model on the $\beta$-plane. Dt. hydrogr. Z., 35: 187-201.

Leetmaa, A. and Bunker, A.F., 1978. Updated charts of the mean annual wind stress, convergences in the Ekman layers and Sverdrup transports in the North Atlantic. J. Mar. Res., 36: 311-322.

Machenhauer, B., 1979. The spectral method. In: Numerical methods used in atmospheric models. GARP Publ. Series No. 17, 2: 124-275.

Marshall, J.C., 1984. Eddy-mean flow interaction in a barotropic ocean model. Q. J. R. Meteorol. Soc., 110: 573-590.

Marshall, J.C. and Shutts, G.J., 1981. A note on rotational and divergent eddy fluxes. J. Phys. Oceanogr., 11: 1677-1680.

Munk, W.H., 1950. On the wind-driven ocean circulation. J. Meteorol., 7: 79-93.

Niiler, P.P., 1966. On the theory of the wind-driven ocean circulation. Deep-Sea Res., 13: 597-606.

Orszag, S.A., 1969. Numerical methods for the simulation of turbulence. Phys. Fluids (Suppl. II), 12: $250-257$.

Robinson, A.R., Harrison, D.E. and Haidvogel, D.B., 1979. Mesoscale eddies and general ocean circulation models. Dyn. Atmos. Oceans, 3: 143-180.

Schmitz, W.J., Jr., 1980. Weakly depth-dependent segments of the North Atlantic circulation. J. Mar. Res., 38: 111-135.

Stommel, H., 1948. The westward intensification of wind-driven ocean currents. Trans. Am. Geophys. Union, 29: 202-206.

Veronis, G., 1963. An analysis of wind-driven ocean circulation with a limited number of Fourier components. J. Atmos. Sci., 20: 577-593.

Veronis, G., 1966a. Wind-driven ocean circulation-Part 1. Linear theory and perturbation analysis. Deep-Sea Res., 13: 17-29.

Veronis, G., 1966b. Wind-driven ocean circulation-Part 2. Numerical solution of the non-linear problem. Deep-Sea Res., 13: 31-55.

Veronis, G., 1966c. Generation of mean ocean circulation by fluctuating winds. Tellus, 18: 67-76.

Worthington, L.V., 1976. On the North Atlantic Circulation. The John Hopkins Oceanographic Studies 6, $110 \mathrm{pp}$. 